Supporting Information for

\title{
Unexpected CNN-to-CC Ligand Rearrangement in Pincer-Ruthenium Pre-catalysts Leads to a Base-Free Catalyst for Ester Hydrogenation
}

Linh Le, Jiachen Liu, Tianyi He, Jack C. Malek, Tia N. Cervarich, John C. Buttner, John Pham, Jason M.

Keith, and Anthony R. Chianese

Department of Chemistry, Colgate University, 13 Oak Drive, Hamilton, NY 13346

\section{Contents}

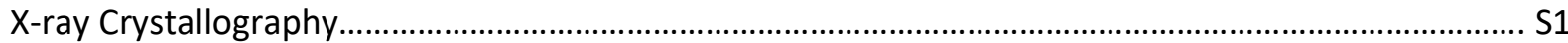

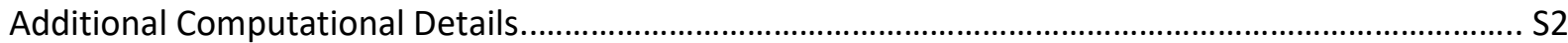

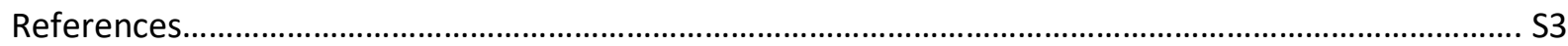

NMR Spectra

X-ray Crystallography, General Methods. Structure determinations were performed on an Oxford Diffraction Gemini-R diffractometer, using Mo-K $\alpha$ radiation at $110 \mathrm{~K}$ unless otherwise noted. Crystals were mounted on Hampton Research Cryoloops using Paratone-N oil. Unit cell determination, data collection and reduction, and empirical absorption correction were performed using the CrysAlisPro software package. ${ }^{1}$ Direct methods structure solution was accomplished using SIR92, ${ }^{2}$ and full-matrix least-squares refinement was carried out using CRYSTALS. ${ }^{3}$ All non-hydrogen atoms were refined anisotropically. Unless otherwise noted, hydrogen atoms were placed in calculated positions, and their positions were initially refined using distance and angle restraints. All hydrogen positions were fixed in place for the final refinement cycles.

$\mathrm{X}$-ray Structure Determination of cis-Ru-dipp-Et-PPh ${ }_{3}$ X-ray quality crystals were grown by slow evaporation of a pentane solution. The ruthenium-bound hydride was located in the difference map, and its position was refined freely before being fixed in place for the final refinement cycles. The NEt ${ }_{2}$ group was disordered over two positions; the major component refined to an occupancy of 0.559 .

X-ray Structure Determination of trans-Ru-Mes-Et-PPh. X-ray quality crystals were grown by slow evaporation of an acetonitrile solution. The ruthenium-bound hydride was located in the difference map, and its position was refined freely before being fixed in place for the final refinement cycles.

X-ray Structure Determination of Ru-Mes-Et-PCу. X-ray quality crystals were grown by slow evaporation of a pentane solution. $\mathrm{Cu}-\mathrm{K} \alpha$ radiation was used. The ruthenium-bound hydride was located in the difference map, and its position was refined freely before being fixed in place for the final 
refinement cycles. A cyclohexyl group was disordered over two positions; the major component refined to an occupancy of 0.677 .

X-ray Structure Determination of Ru-dipp-Me-PCу. X-ray quality crystals were grown by layering acetonitrile over a concentrated solution in dichloromethane. The ruthenium-bound hydride was located in the difference map, and its position was refined freely before being fixed in place for the final refinement cycles. A cyclohexyl group was disordered over two positions; the major component refined to an occupancy of 0.676 . Two molecules of dichloromethane were present in the asymmetric unit. An A-level alert was generated by CheckCIF due to a short intramolecular contact between $\mathrm{H} 241$ and $\mathrm{H} 693$. $\mathrm{H} 241$ is connected to a disordered carbon on a cyclohexyl group, and $\mathrm{H} 693$ is connected to a nearby hydrogen on an isopropyl group, which likely has a minor disorder that we were not able to model.

X-ray Structure Determination of Ru-dipp-i $\mathrm{Pr}^{-} \mathrm{PC}_{\mathbf{3}} . \mathrm{X}$-ray quality crystals were grown by layering acetonitrile over a concentrated solution in dichloromethane. $\mathrm{Cu}-\mathrm{K} \alpha$ radiation was used. The rutheniumbound hydride was located in the difference map, and its position was refined freely before being fixed in place for the final refinement cycles. A cyclohexyl group was disordered over two positions; the major component refined to an occupancy of 0.800 . An A-level alert was generated by CheckCIF due to a short intramolecular contact between $\mathrm{H} 12$ and $\mathrm{H} 663 . \mathrm{H} 12$ is connected to a disordered carbon on a cyclohexyl group, and $\mathrm{H} 663$ is connected to a nearby hydrogen on an isopropyl group, which likely has a minor disorder that we were not able to model.

Additional Computational Details. All DFT calculations were initially performed as described in the main text, with thermal corrections to the free energies calculated at $298.15 \mathrm{~K}$. The correction of the standard state from $1 \mathrm{~atm}$ to $1 \mathrm{M}$, added to each species, is calculated as $R_{1} T \ln \left(R_{2} T\right)$, where $R_{1}=8.31447 \mathrm{~J} /(\mathrm{mol} \cdot \mathrm{K})$ and $R_{2}=0.08206 \mathrm{~L} \cdot \mathrm{atm} /(\mathrm{mol} \cdot \mathrm{K})$. At $298.15 \mathrm{~K}$, this correction is $1.89 \mathrm{kcal} / \mathrm{mol}$.

To assess the feasibility of the conversion of Ru-dipp-Et-PC $\mathbf{y}_{\mathbf{3}}$ back to $\mathrm{B}$ under the conditions of catalysis at $105^{\circ} \mathrm{C}$ or $378.15 \mathrm{~K}$, the thermal corrections to the free energies were recalculated at this temperature. At $378.15 \mathrm{~K}$, the correction of the standard state from $1 \mathrm{~atm}$ to $1 \mathrm{M}$, added to each species, is $2.58 \mathrm{kcal} / \mathrm{mol}$.

Table S1 shows the electronic energies and thermal corrections at both temperatures, and shows the relative standard-state-corrected free energies of each species at $298.15 \mathrm{~K}$ and $378.15 \mathrm{~K}$. The free energies at $298.15 \mathrm{~K}$, shown in Chart 2 and Figure 6 of the main text, are referenced against B $+2 \mathrm{PCy}_{3}$. The free energies at $378.15 \mathrm{~K}$ are referenced against Ru-dipp-Et-PC $\mathbf{Y}_{\mathbf{3}}$, to demonstrate the kinetic and thermodynamic reversibility of the reaction at the operating temperature of the catalytic reactions. At $378.15 \mathrm{~K}$, the conversion of Ru-dipp-Et-PCy $\mathbf{y}_{3}$ to $\mathbf{B}$ is calculated to have a highest barrier of $23.5 \mathrm{kcal} / \mathrm{mol}$ (through CD-TS), and an overall free energy change of $+5.9 \mathrm{kcal} / \mathrm{mol}$. 
Table S1. Calculated electronic and free energies for all reported species, at $298.15 \mathrm{~K}$ and $378.15 \mathrm{~K}$. Relative free energies include a correction to the $1 \mathrm{M}$ standard state.

\section{Energies of isolated species}

\begin{tabular}{llll} 
Compound & $\begin{array}{l}\text { Electronic energy } \\
\text { (hartrees) }\end{array}$ & $\begin{array}{l}\text { Free energy correction } \\
\text { (hartrees) } \\
298.15 \mathrm{~K}\end{array}$ & $378.15 \mathrm{~K}$ \\
\hline $\mathrm{PCy}_{3}$ & -1047.457236 & 0.435914 & 0.416452 \\
$\mathrm{~A}$ & -1553.568788 & 0.536076 & 0.505810 \\
A-dearom & -1553.562295 & 0.532877 & 0.502771 \\
B & -2601.069071 & 1.004541 & 0.962374 \\
B-dearom & -2601.057916 & 1.003148 & 0.961364 \\
C & -2601.053475 & 0.996339 & 0.952200 \\
CD-TS & -2601.024612 & 0.990858 & 0.945933 \\
D & -2601.034482 & 0.993430 & 0.948910 \\
DE-TS & -2601.027645 & 0.990062 & 0.945843 \\
E & -2601.059367 & 0.989785 & 0.944761 \\
EP-TS & -3648.519605 & 1.455151 & 1.397999 \\
Ru-dipp-Et-PCу 3 & -3648.563868 & 1.466236 & 1.411076
\end{tabular}

\section{Energies with mass balance}

\begin{tabular}{|c|c|c|c|c|c|}
\hline \multirow[b]{2}{*}{ Compound(s) } & \multirow{2}{*}{$\begin{array}{l}\text { Electronic energy } \\
\text { (hartrees) }\end{array}$} & \multicolumn{2}{|c|}{$\begin{array}{l}\text { Free energy correction } \\
\text { (hartrees) }\end{array}$} & \multicolumn{2}{|c|}{$\begin{array}{l}\text { Relative free energy at } \\
1 \mathrm{M} \text { std. state }(\mathrm{kcal} / \mathrm{mol})\end{array}$} \\
\hline & & $298.15 \mathrm{~K}$ & $378.15 \mathrm{~K}$ & $298.15 \mathrm{~K}$ & $378.15 \mathrm{~K}$ \\
\hline$A+2 P C y_{3}$ & -3648.483260 & 1.407904 & 1.338714 & 8.5 & 10.3 \\
\hline A-dearom + 2 РСуз & -3648.476767 & 1.404705 & 1.335675 & 10.5 & 12.5 \\
\hline $\mathrm{B}+\mathrm{PC}_{3}$ & -3648.526307 & 1.440455 & 1.378826 & 0.0 & 5.9 \\
\hline B-dearom + РСуз & -3648.515152 & 1.439062 & 1.377816 & 6.1 & 12.3 \\
\hline С + РСуз & -3648.510711 & 1.432253 & 1.368652 & 4.6 & 9.3 \\
\hline CD-TS + PCуз & -3648.481848 & 1.426772 & 1.362385 & 19.3 & 23.5 \\
\hline $\mathrm{D}+\mathrm{PC}_{3}$ & -3648.491718 & 1.429344 & 1.365362 & 14.7 & 19.2 \\
\hline DE-TS + PCуз & -3648.484881 & 1.425976 & 1.362295 & 16.9 & 21.5 \\
\hline $\begin{array}{l}\mathrm{E}+\mathrm{PC} \mathrm{y}_{3} \\
\text { EP-TS }\end{array}$ & $\begin{array}{l}-3648.516603 \\
-3648.519605\end{array}$ & $\begin{array}{l}1.425699 \\
1.455151\end{array}$ & $\begin{array}{l}1.361213 \\
1.397999\end{array}$ & $\begin{array}{l}-3.2 \\
11.5\end{array}$ & $\begin{array}{l}0.9 \\
19.6\end{array}$ \\
\hline Ru-dipp-Et-PCуз & -3648.563868 & 1.466236 & 1.411076 & -9.3 & 0.0 \\
\hline
\end{tabular}

\section{References}

(1) Oxford Diffraction (2007). Oxford Diffraction Ltd., Xcalibur Ccd System, Crysalispro Software System, Version 1.171.32.

(2) Altomare, A.; Cascarano, M.; Giacovazzo, C.; Guagliardi, A.; Burla, M. C.; Polidori, G.; Camalli, M. Sir92 - a Program for Automatic Solution of Crystal Structures by Direct Methods. J. Appl. Cryst. 1994, 27, 435-436.

(3) Betteridge, P. W.; Carruthers, J. R.; Cooper, R. I.; Prout, K.; Watkin, D. J. Crystals. J. Appl. Cryst. 2003, $36,1487$. 
NMR Spectra of Ruthenium Complexes
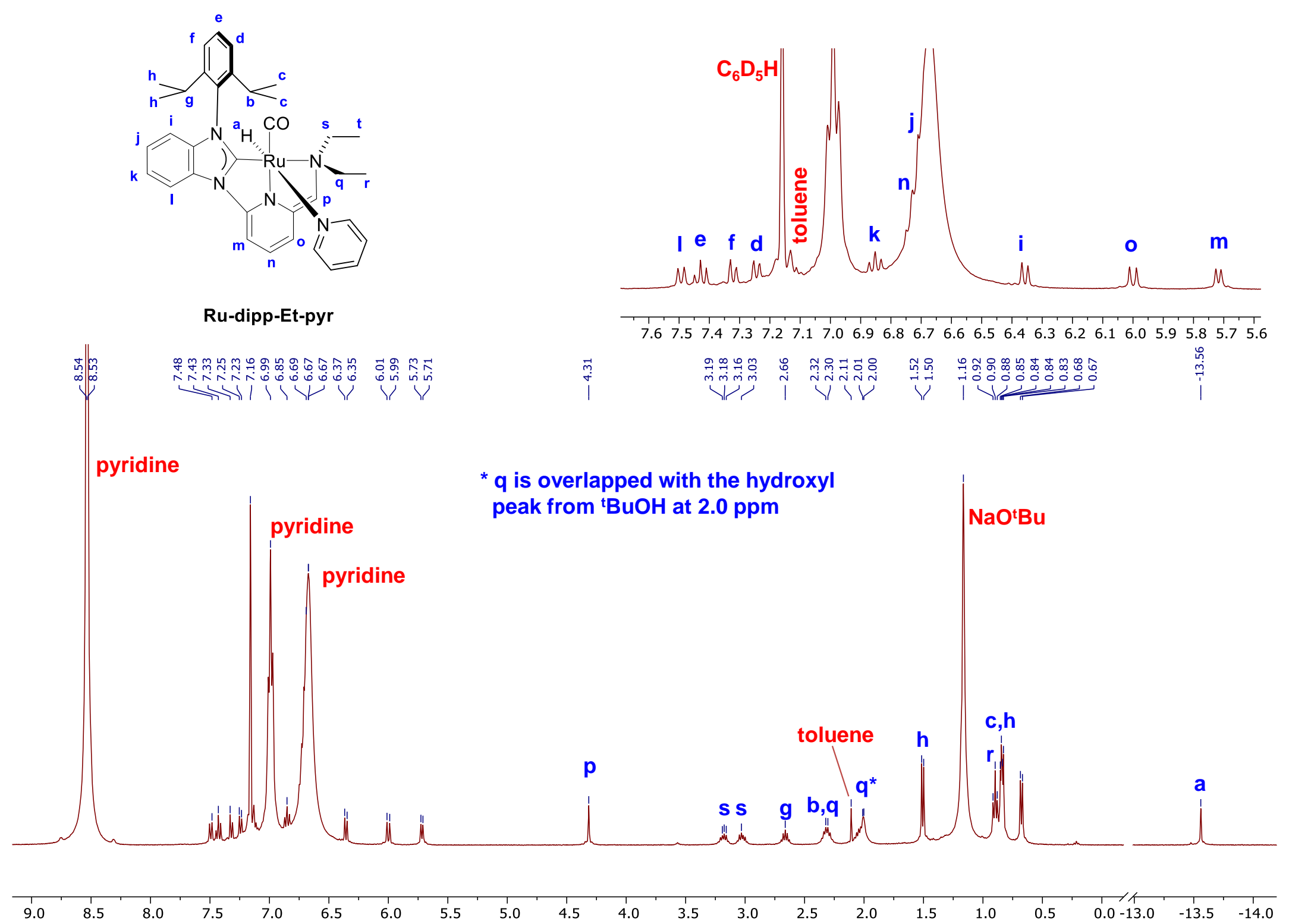

Figure S1. ${ }^{1} \mathrm{H}$ NMR spectrum of Ru-dipp-Et-pyr, as prepared in solution with excess $\mathrm{NaO} \mathrm{O}^{\mathrm{t}} \mathrm{Bu}$ and pyridine. 


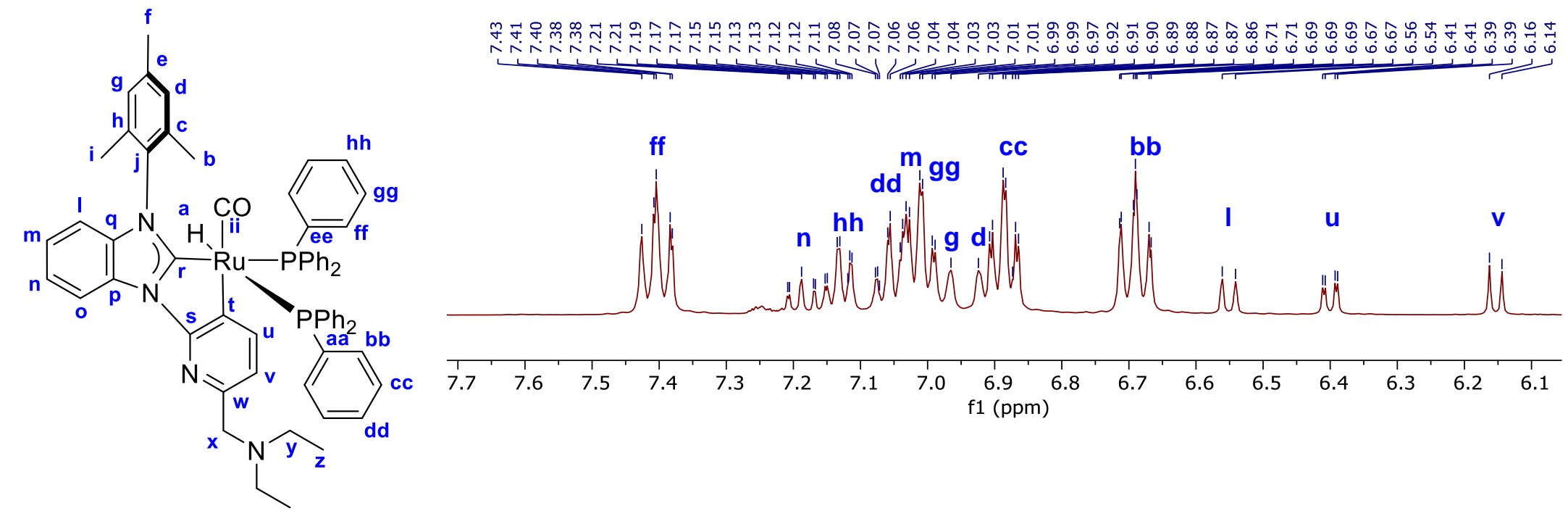

\section{cis-Ru-Mes-Et-PPh}

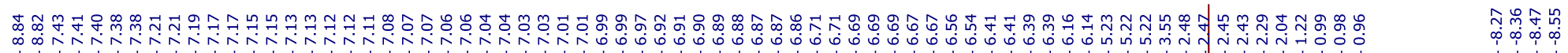

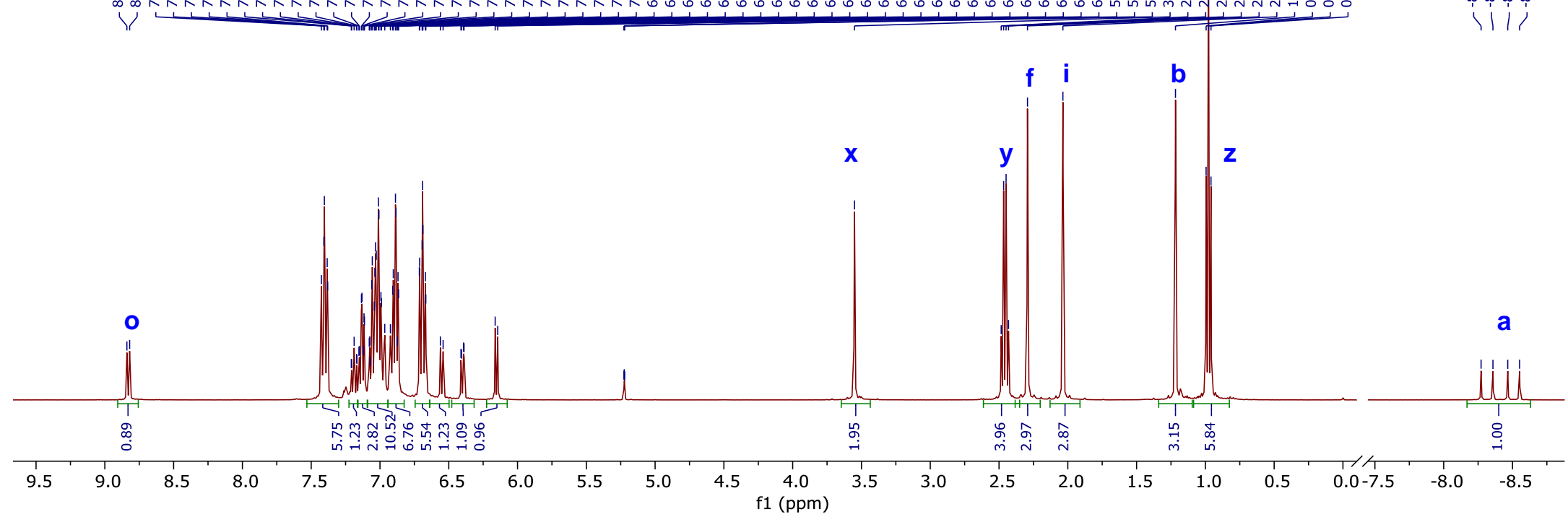

Figure S2. ${ }^{1} \mathrm{H}$ NMR spectrum of cis-Ru-Mes-Et-PPh ${ }_{3}$. 

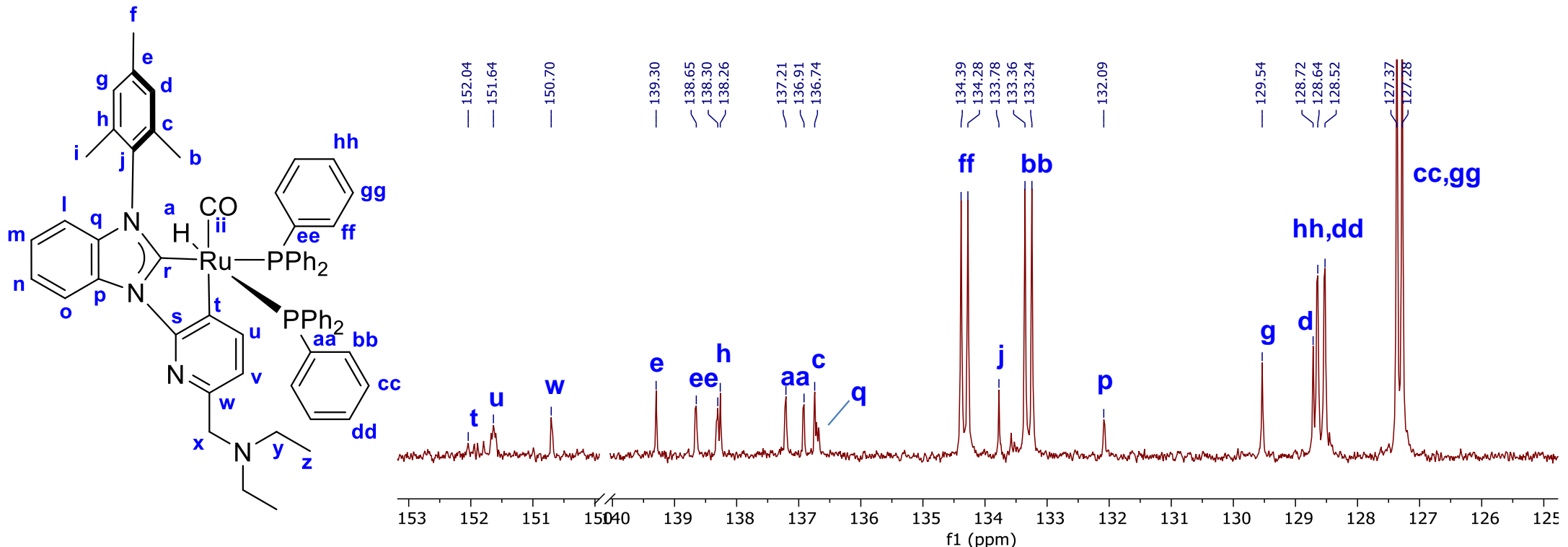

cis-Ru-Mes-Et-PPh

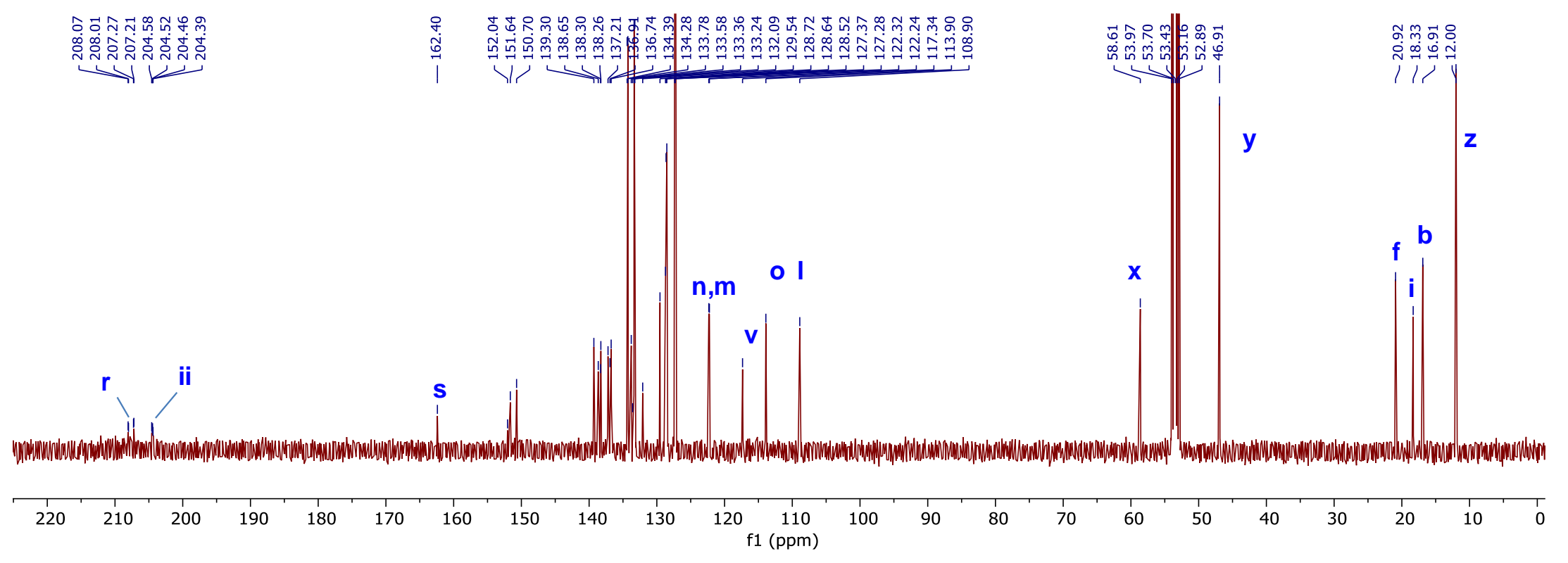

Figure S3. ${ }^{13} \mathrm{C}\left\{{ }^{1} \mathrm{H}\right\}$ NMR spectrum of cis-Ru-Mes-Et-PPh $\mathbf{h}_{3}$ 

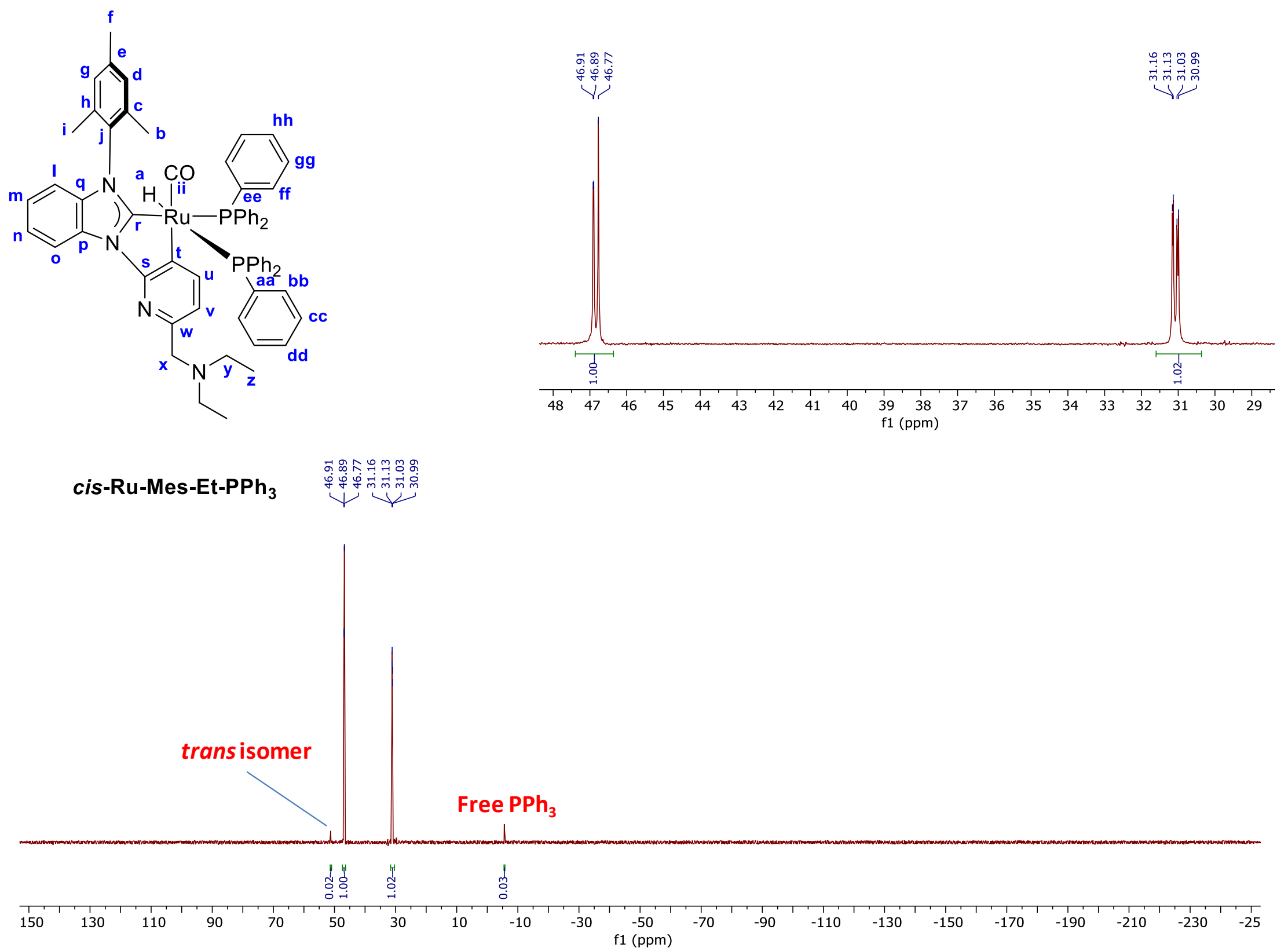

Figure S4. ${ }^{31} \mathrm{P}\left\{{ }^{1} \mathrm{H}\right\}$ NMR spectrum of cis-Ru-Mes-Et-PPh ${ }_{\mathbf{3}}$. 


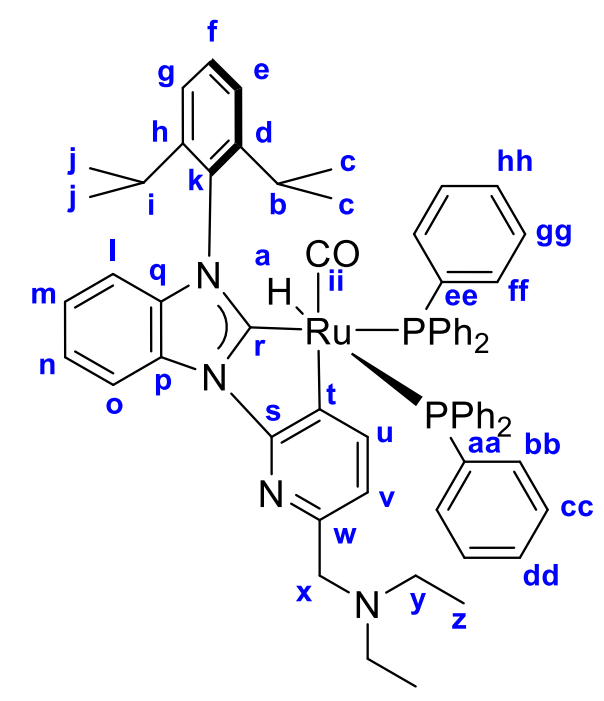

cis-Ru-dipp-Et-PPh

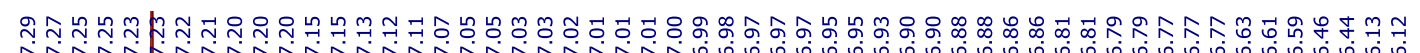
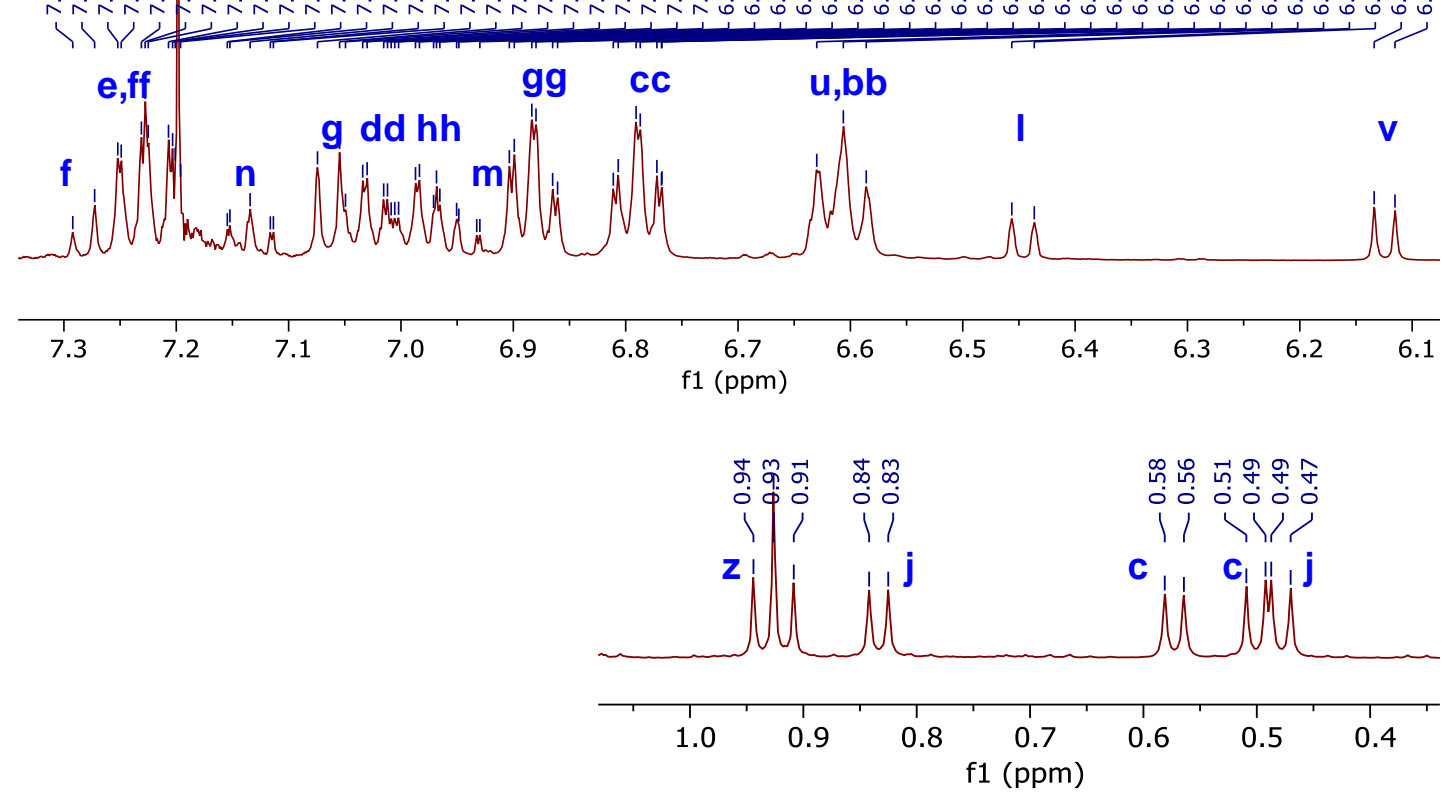

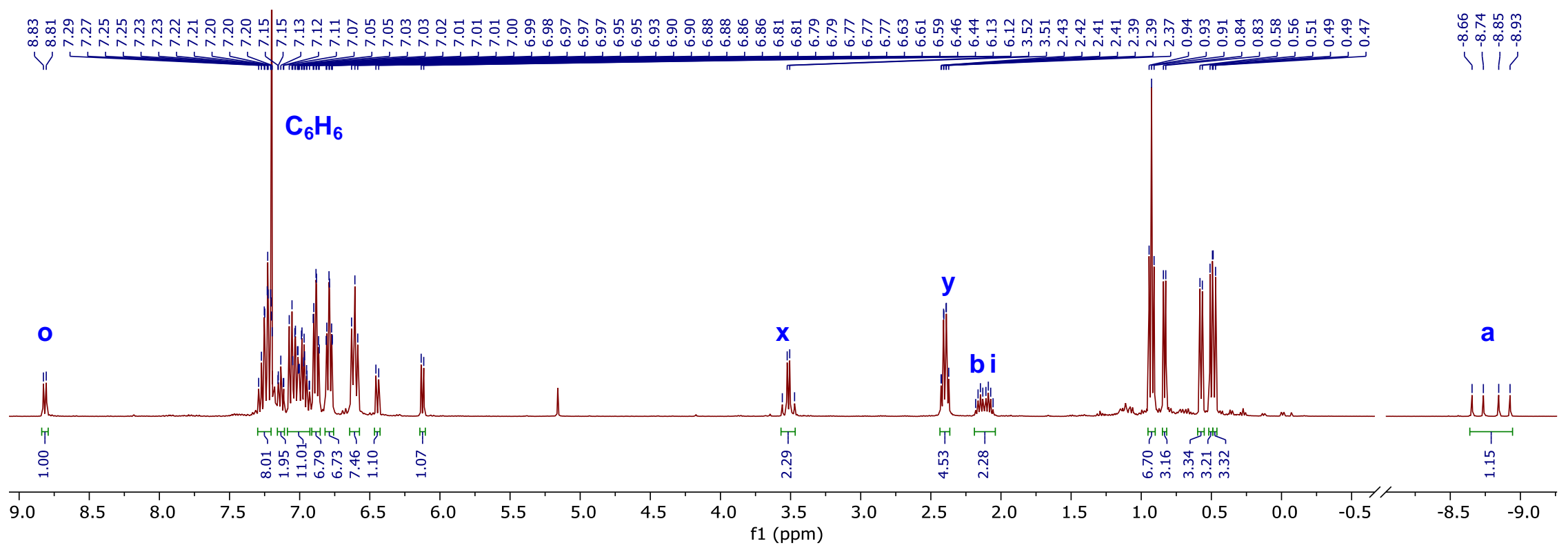

Figure S5. ${ }^{1} \mathrm{H}$ NMR spectrum of cis-Ru-dipp-Et-PPh ${ }_{3}$. 

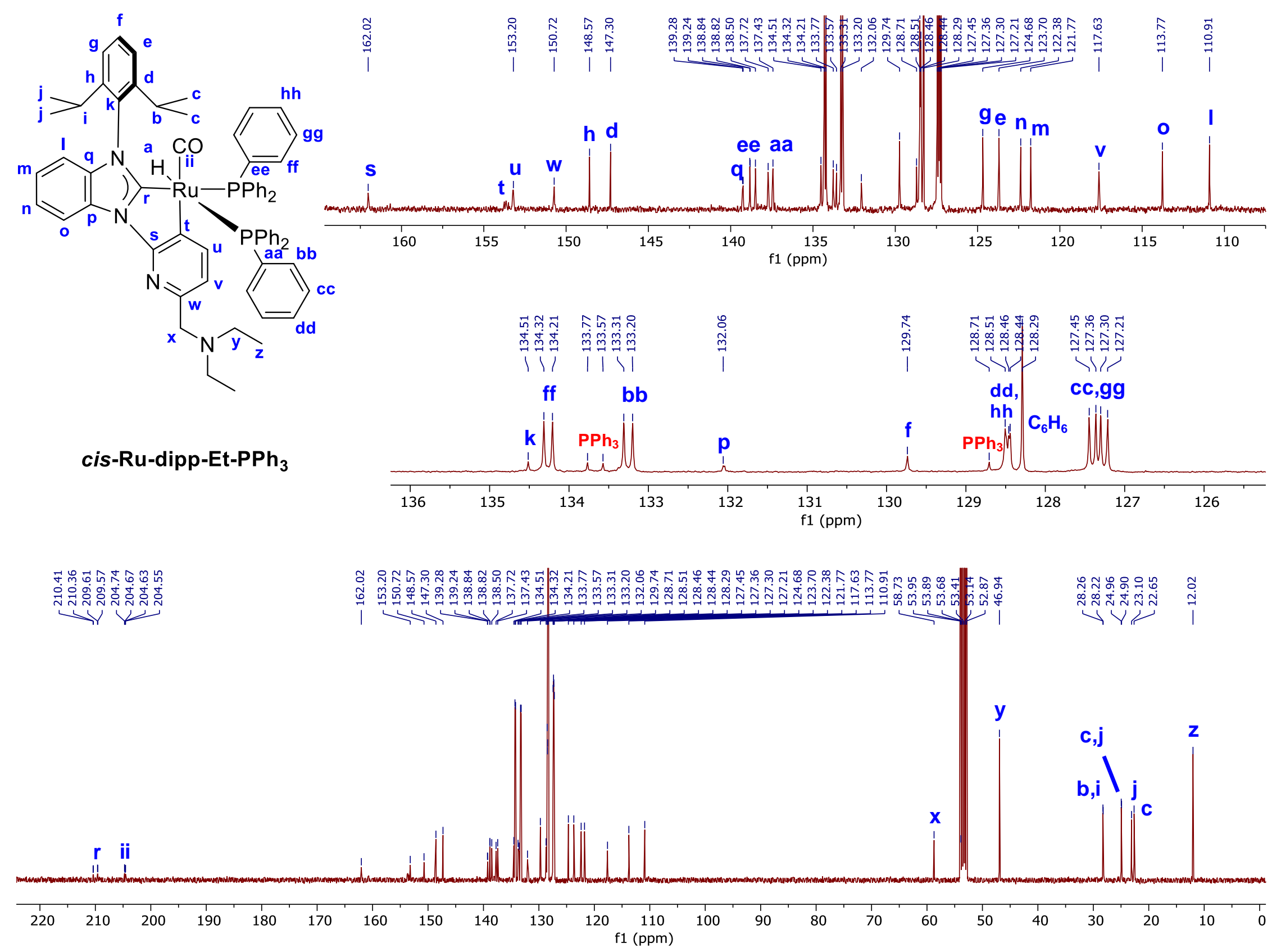

Figure S6. ${ }^{13} \mathrm{C}\left\{{ }^{1} \mathrm{H}\right\}$ NMR spectrum of cis-Ru-dipp-Et-PPh${ }_{\mathbf{3}}$. 

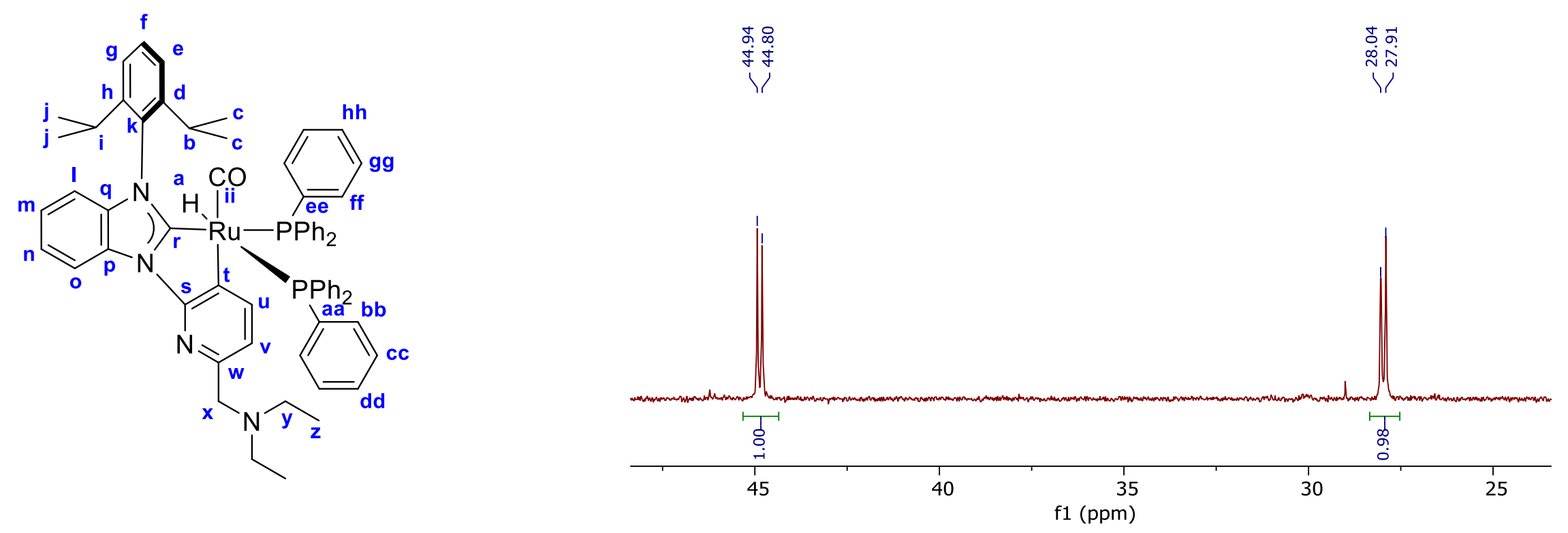

cis-Ru-dipp-Et-PPh ${ }_{3}$
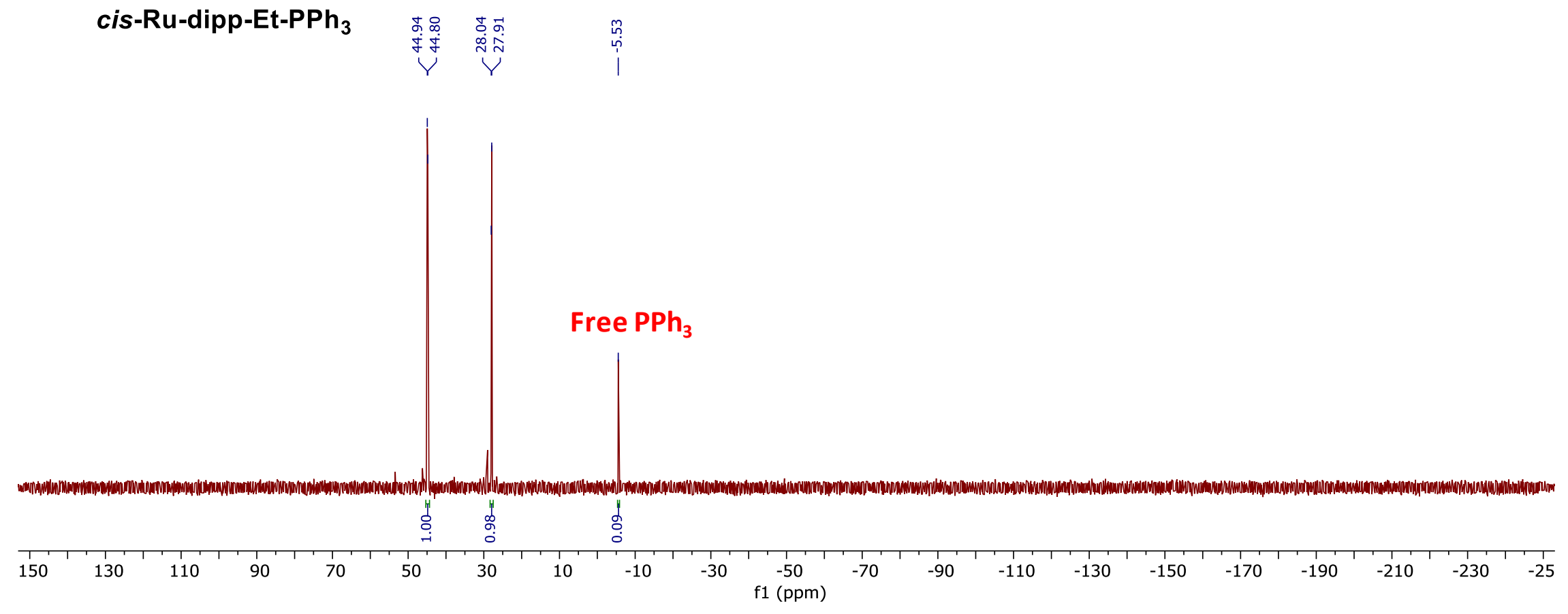

Figure S7. ${ }^{31} \mathrm{P}\left\{{ }^{1} \mathrm{H}\right\}$ NMR spectrum of cis-Ru-dipp-Et-PPh. 


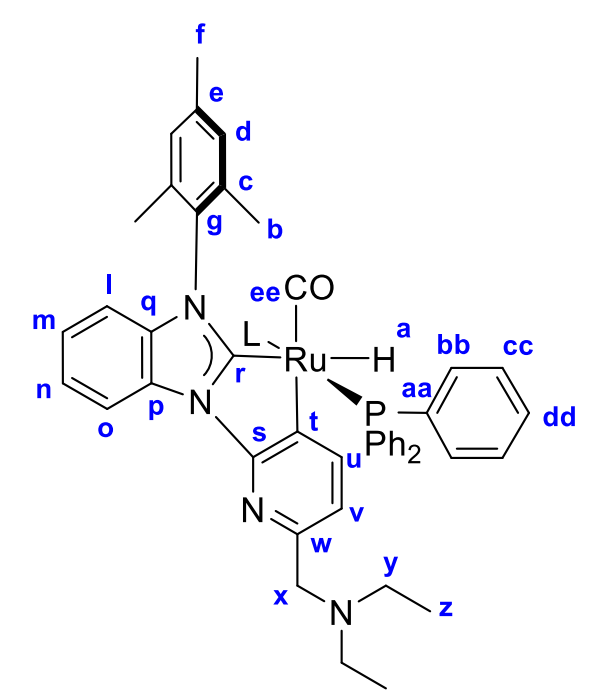

trans-Ru-Mes-Et-PPh

$\mathrm{L}=\mathrm{PPh}_{3}$

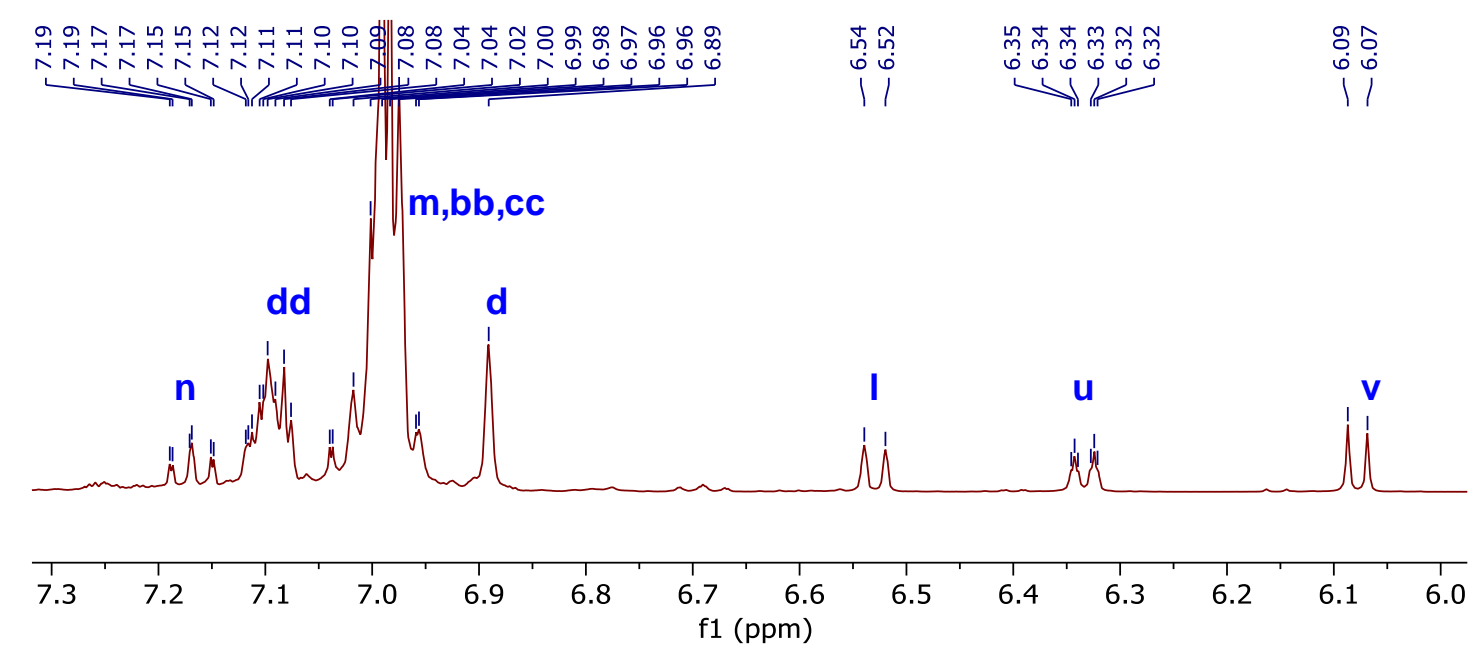

* denotes unidentified impurities

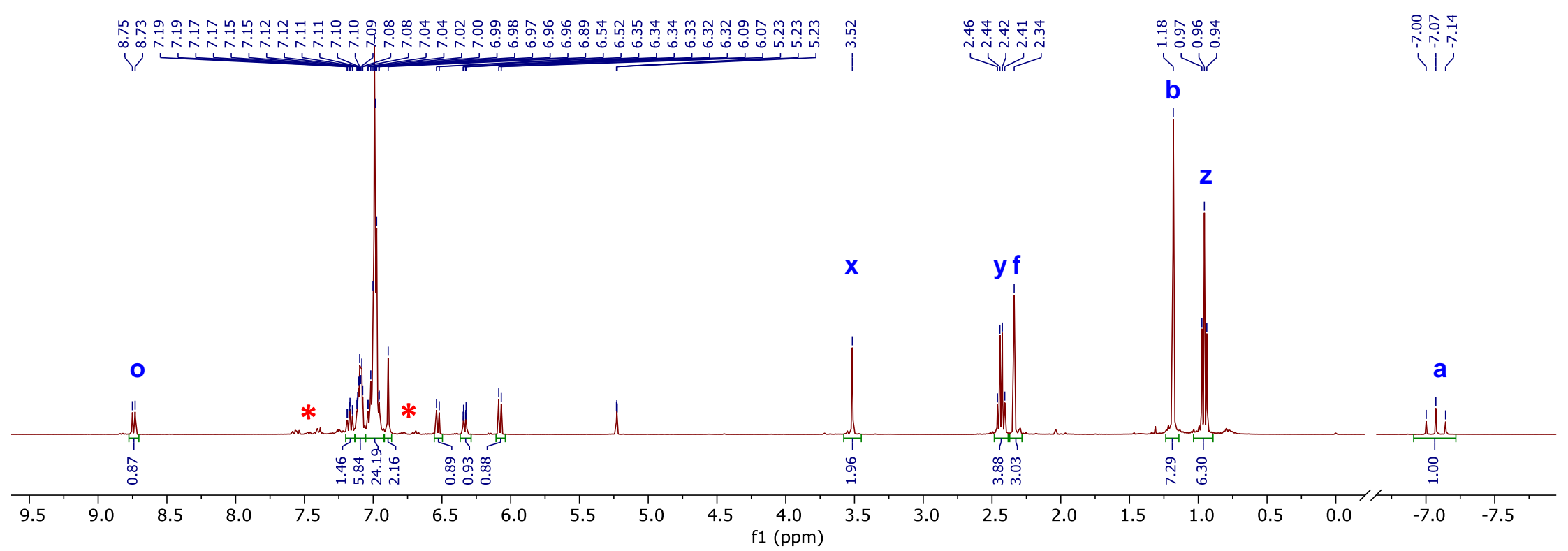

Figure S8. ${ }^{1} \mathrm{H}$ NMR spectrum of trans-Ru-Mes-Et-PPh ${ }_{3}$. 


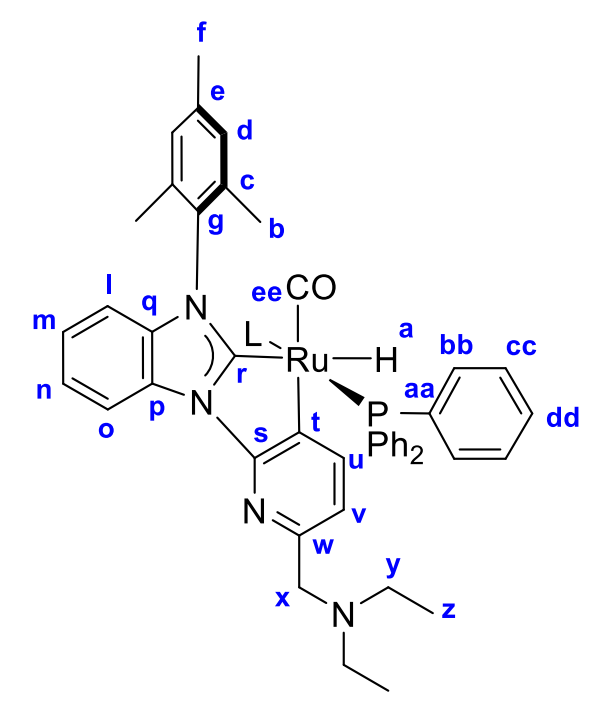

trans-Ru-Mes-Et-PPh $\mathrm{L}=\mathrm{PPh}_{3}$

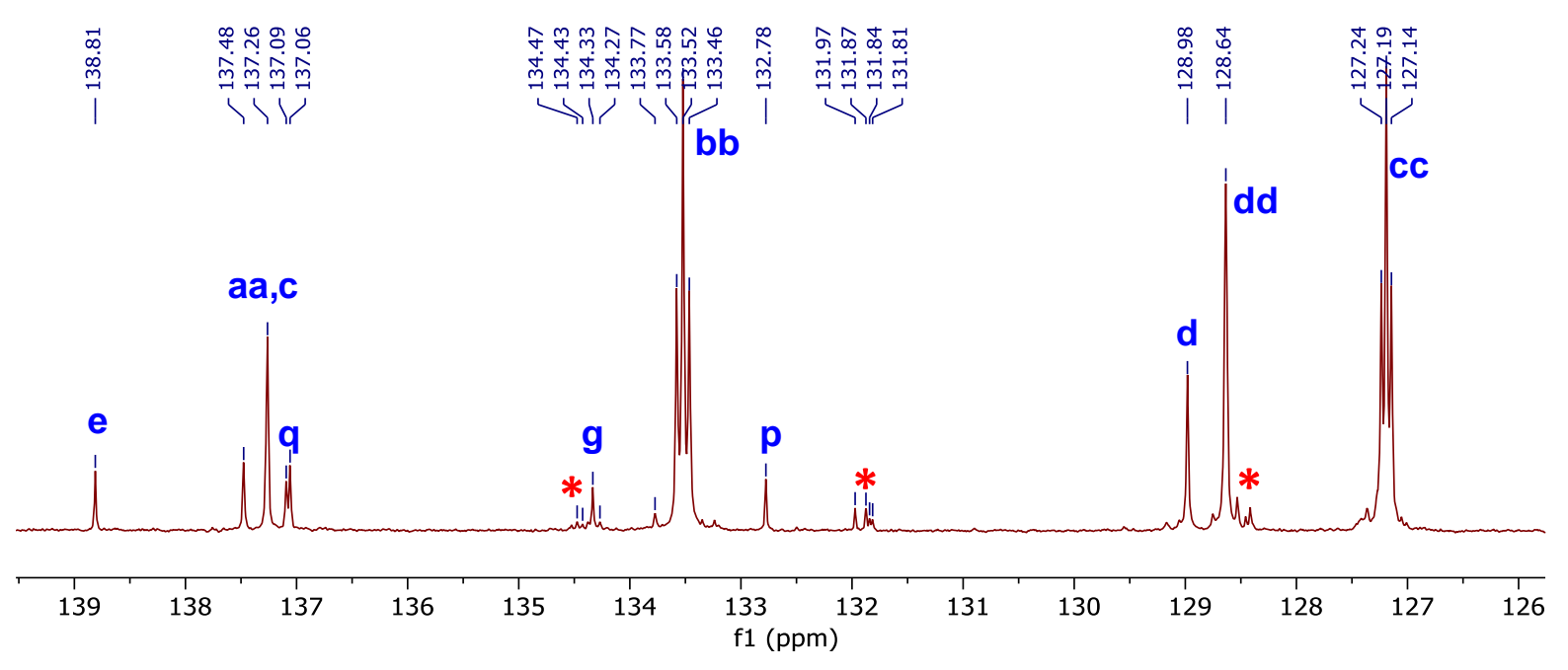

* denotes unidentified impurities

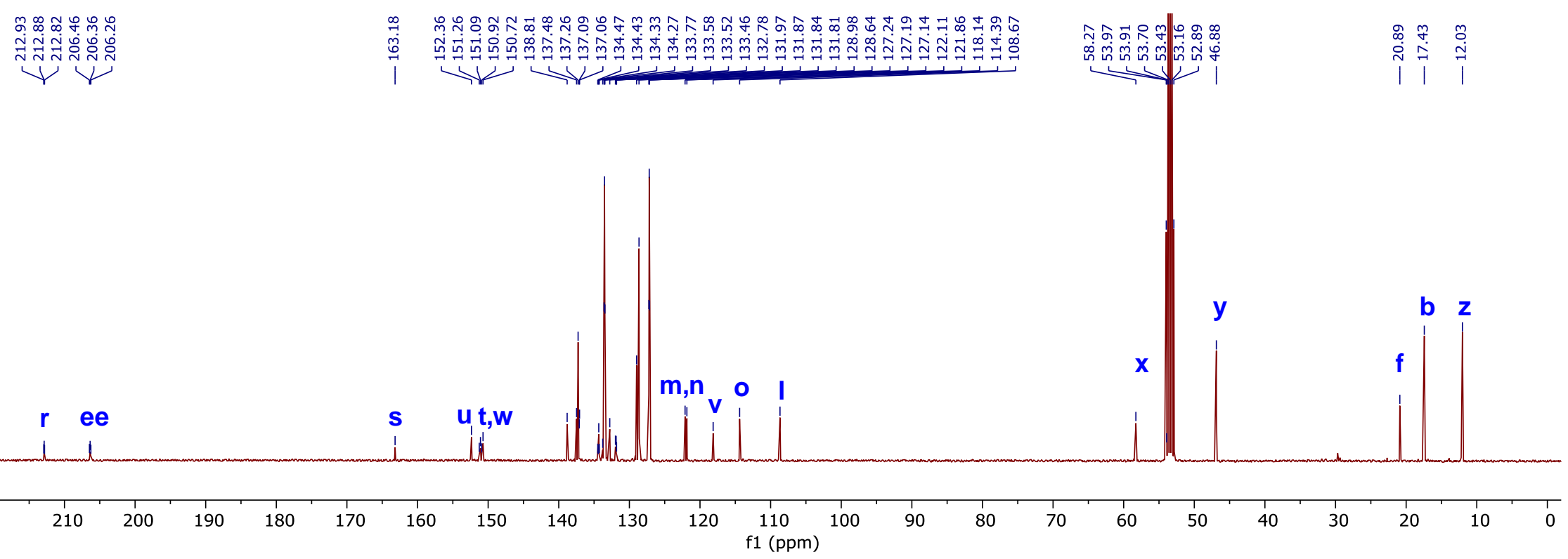

Figure S9. ${ }^{13} \mathrm{C}\left\{{ }^{1} \mathrm{H}\right\}$ NMR spectrum of trans-Ru-Mes-Et-PPh ${ }_{\mathbf{3}}$. 


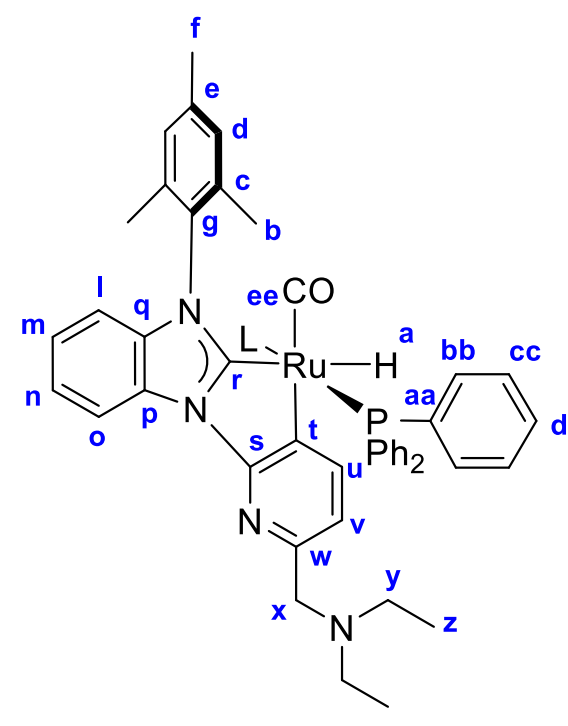

trans-Ru-Mes-Et-PPh

$$
\mathrm{L}=\mathrm{PPh}_{3}
$$

\section{Unknown}

impurities

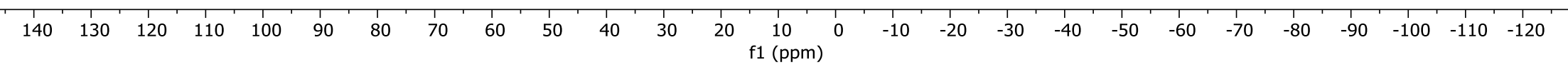

Figure S10. ${ }^{31} \mathrm{P}\left\{{ }^{1} \mathrm{H}\right\}$ NMR spectrum of trans-Ru-Mes-Et-PPh. 


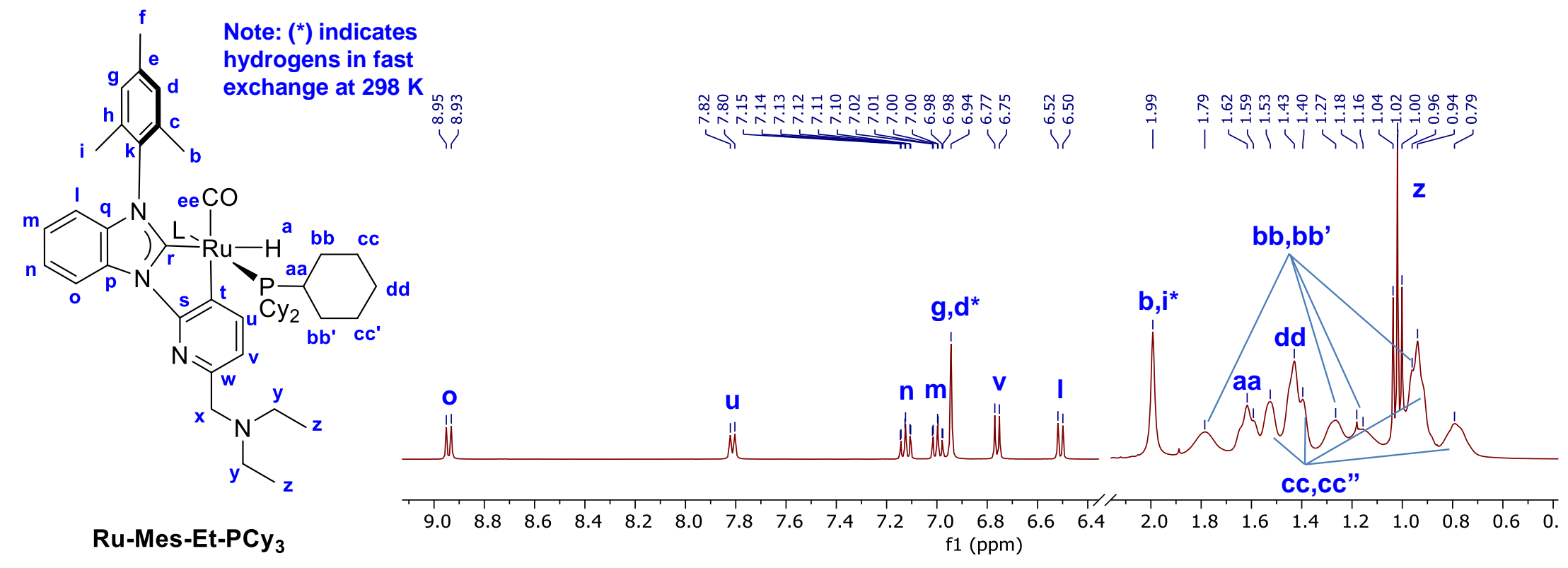

$L=P C y_{3}$

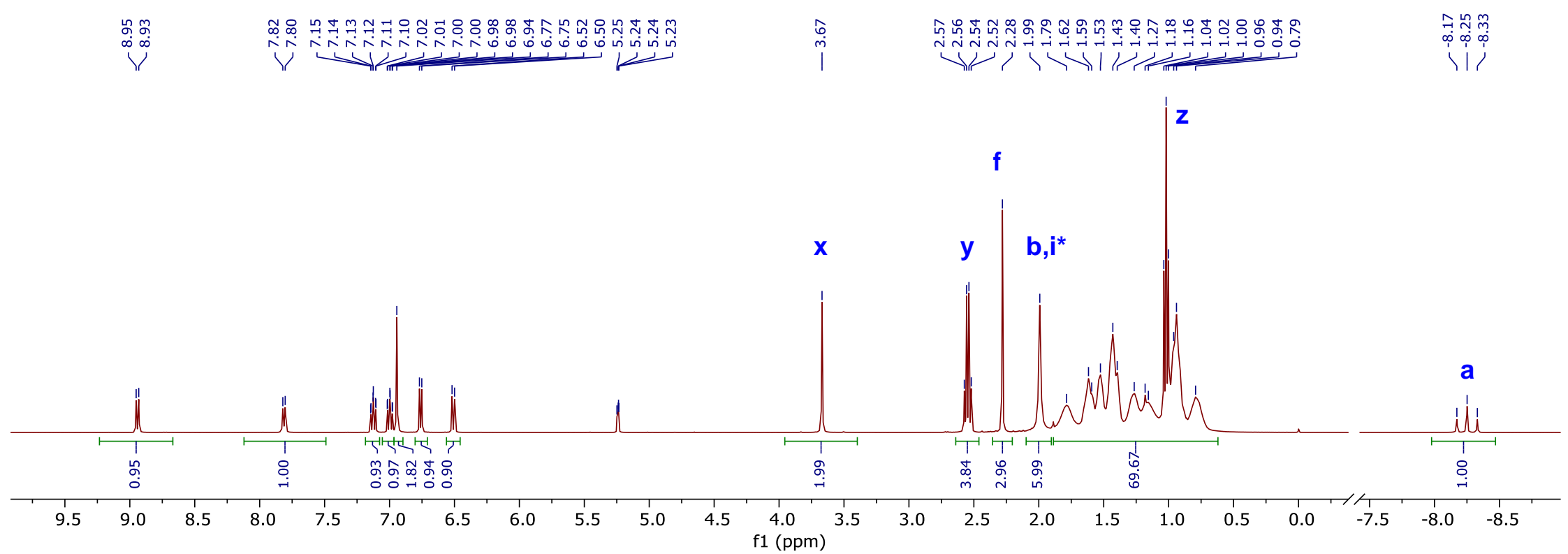

Figure S11. ${ }^{1} \mathrm{H}$ NMR spectrum of Ru-Mes-Et-PCy $\mathbf{y}_{3}$ 


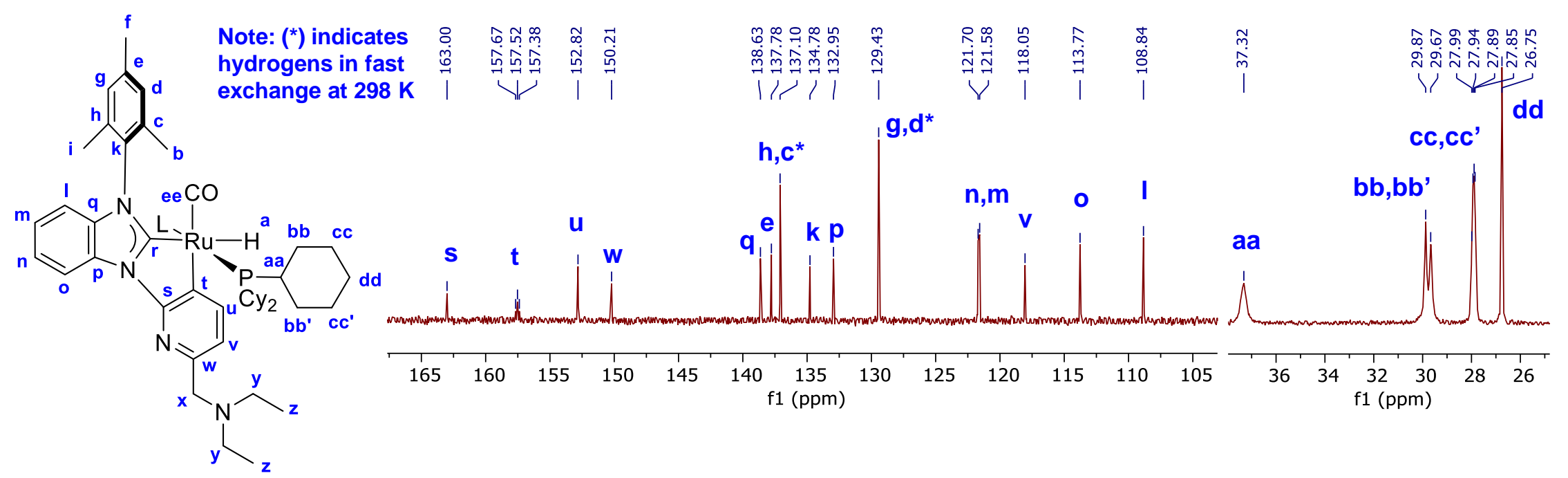

\section{Ru-Mes-Et-PCy $\mathbf{y}_{\mathbf{3}}$}

$L=P C y_{3}$

în

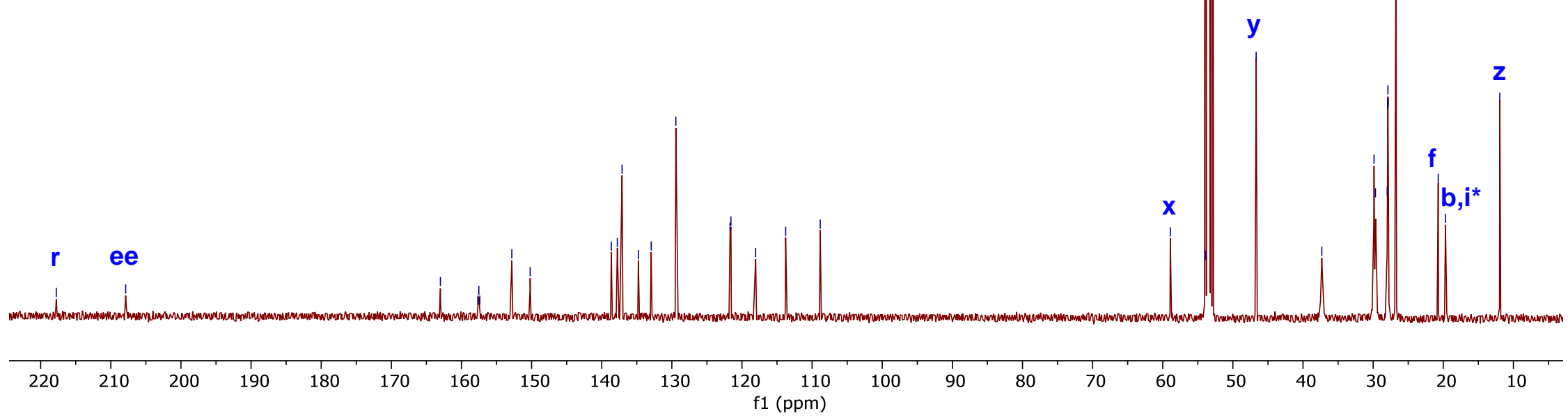

Figure S12. ${ }^{13} \mathrm{C}\left\{{ }^{1} \mathrm{H}\right\}$ NMR spectrum of Ru-Mes-Et-PCy $\mathbf{y}_{3}$. 


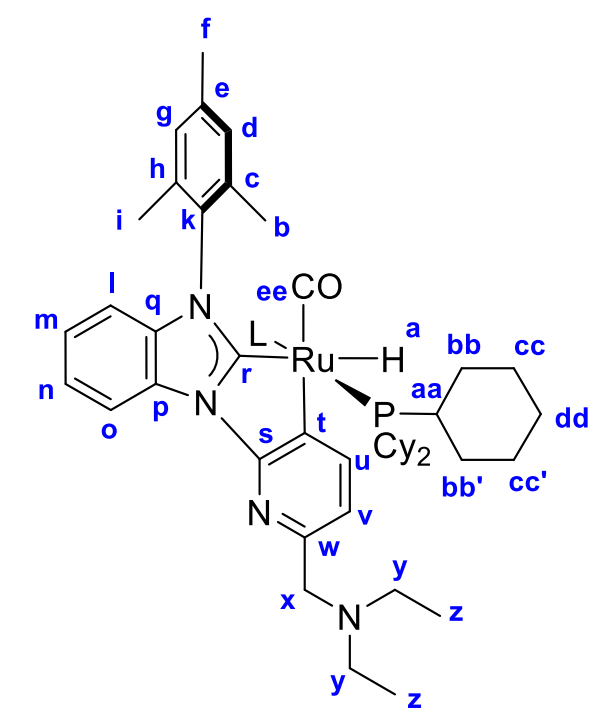

Ru-Mes-Et-PCy $\mathbf{y}_{\mathbf{3}}$

$$
\mathrm{L}=\mathrm{PCy}_{3}
$$

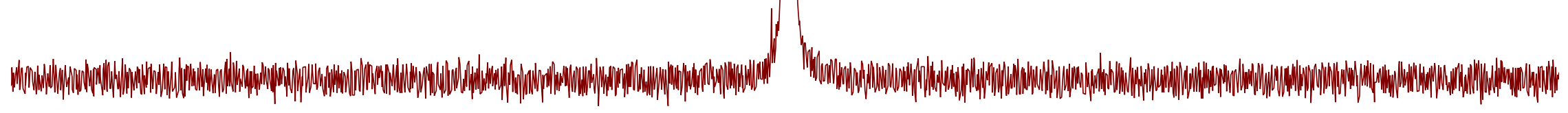

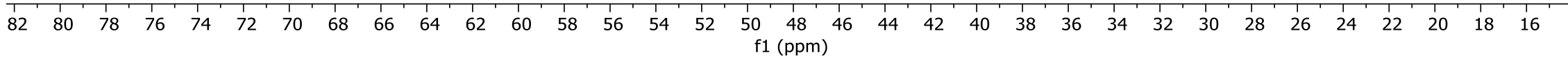

Figure S13. ${ }^{13} \mathrm{C}\left\{{ }^{1} \mathrm{H}\right\}$ NMR spectrum of Ru-Mes-Et-PCy. 


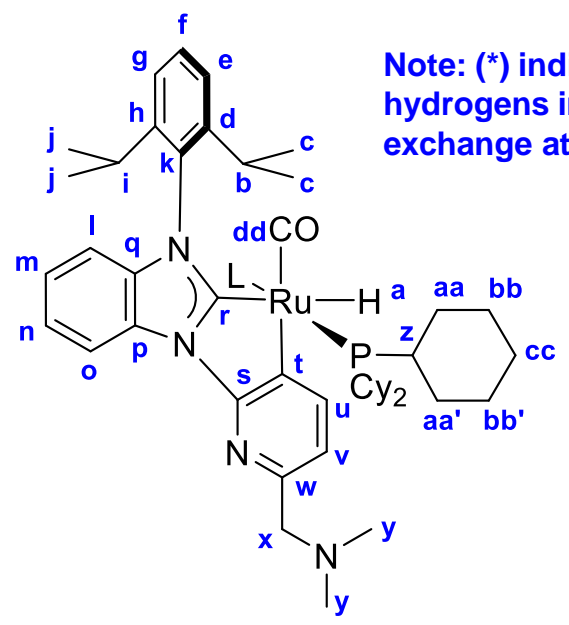

\section{Ru-dipp-Me-PCy $y_{3}$}

$\mathrm{L}=\mathrm{PCy}_{3}$

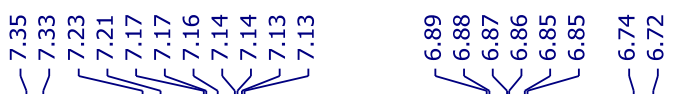

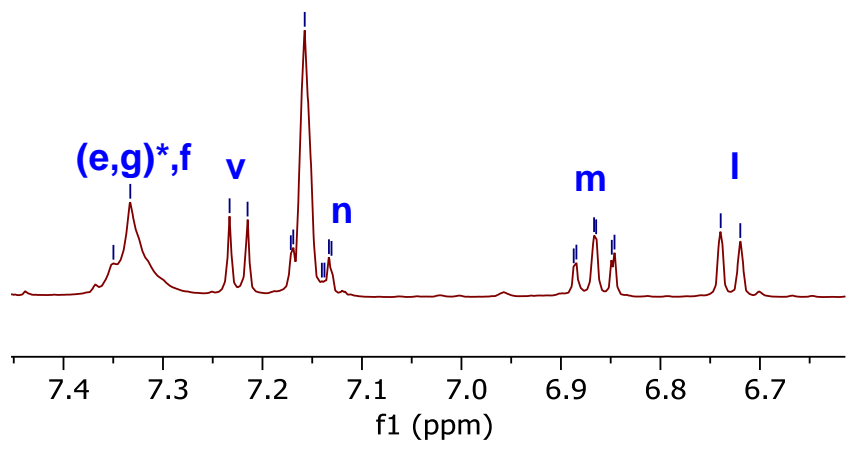

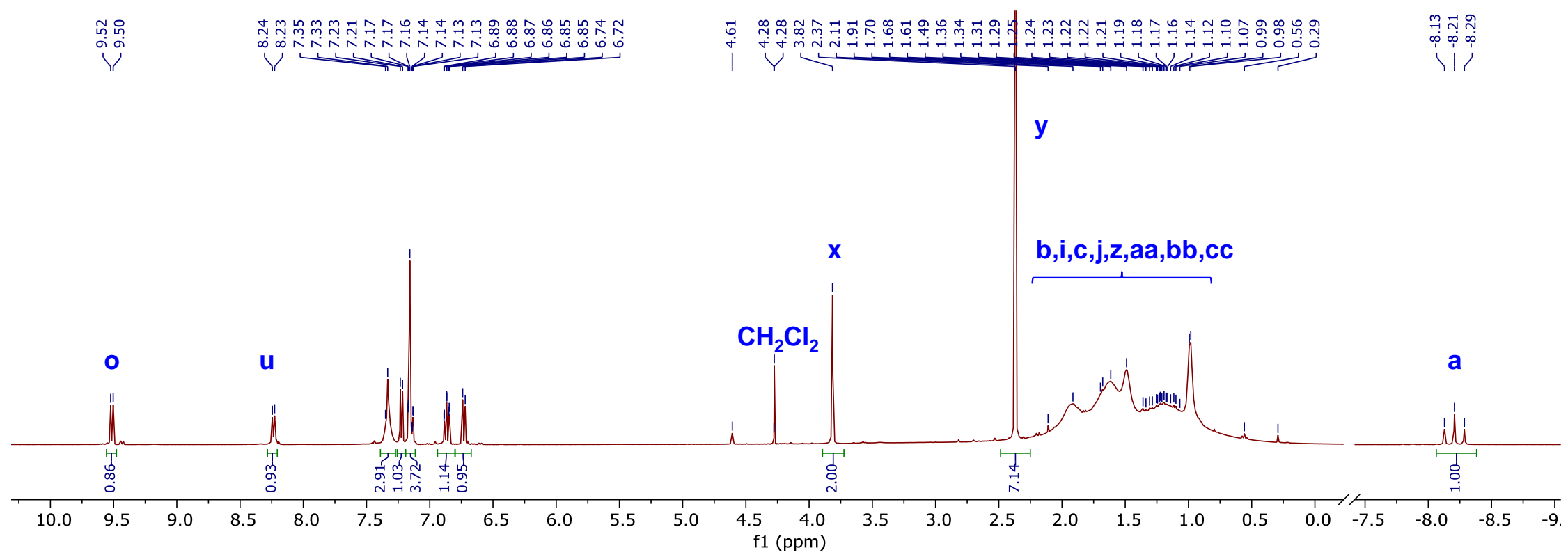

Figure S14. ${ }^{1} \mathrm{H}$ NMR spectrum of Ru-dipp-Me-PCy. 

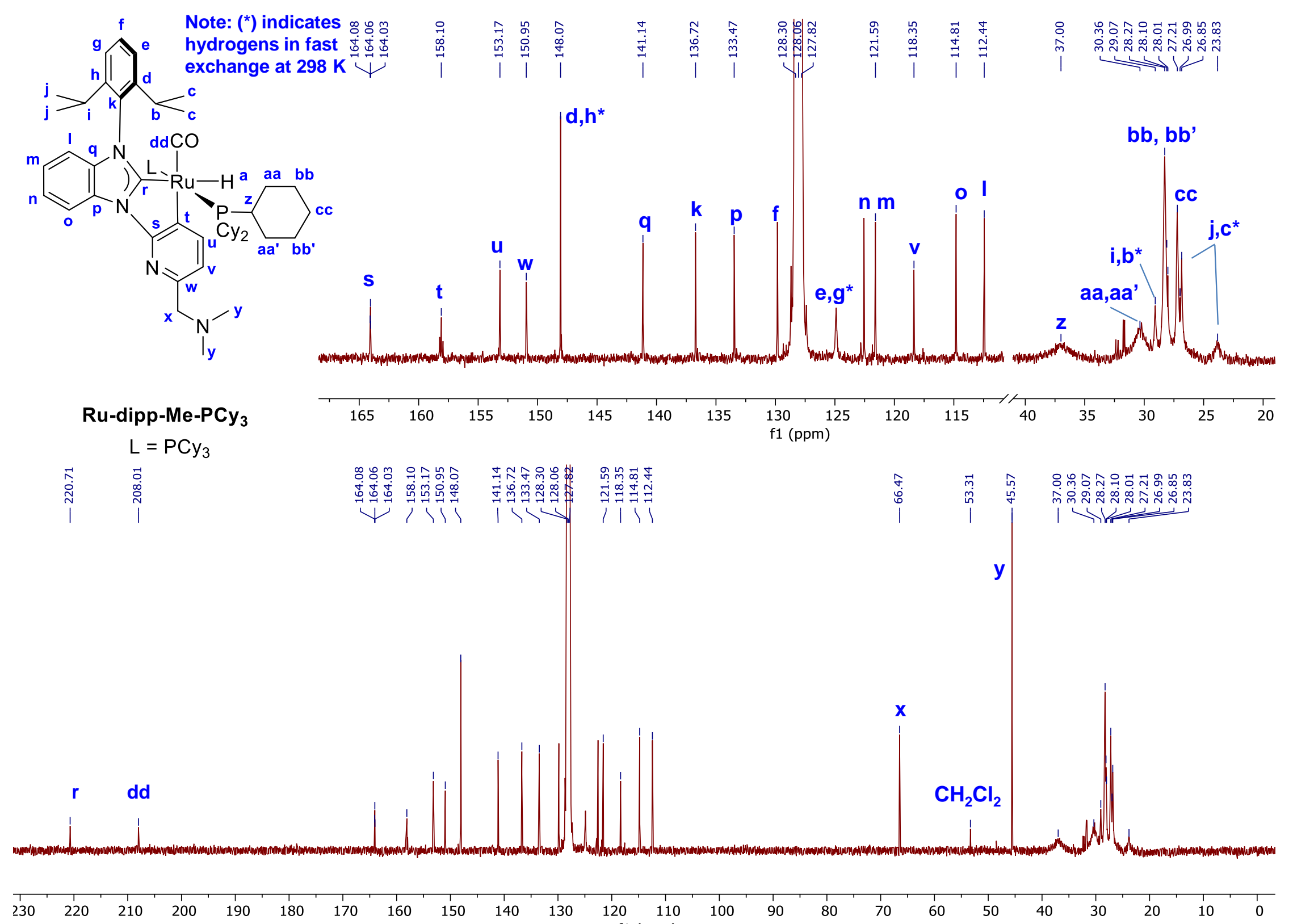

Figure S15. ${ }^{13} \mathrm{C}\left\{{ }^{1} \mathrm{H}\right\}$ NMR spectrum of Ru-dipp-Me-PCy ${ }_{3}$. 


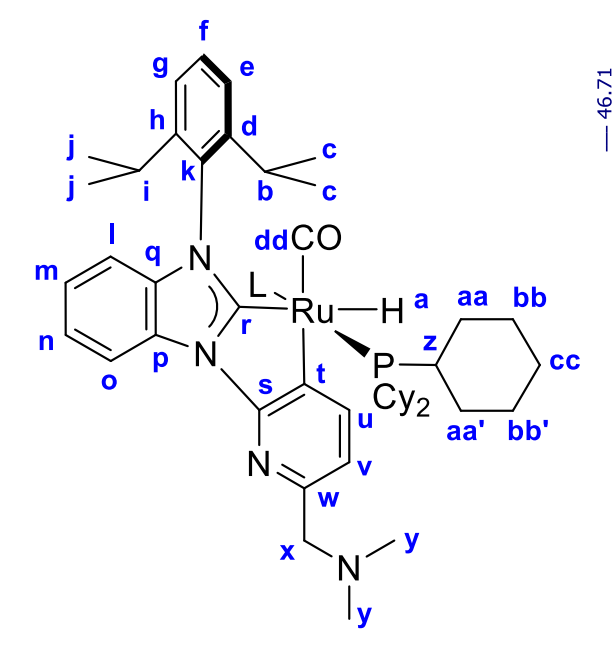

\section{Ru-dipp-Me-PCy ${ }_{3}$}

$$
\mathrm{L}=\mathrm{PCy}_{3}
$$

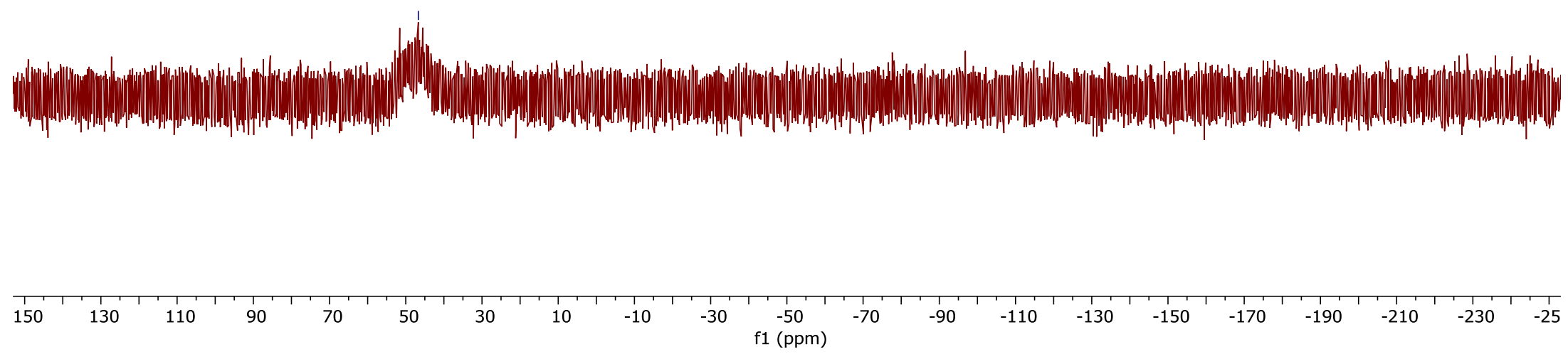

Figure S16. ${ }^{31} \mathrm{P}\left\{{ }^{1} \mathrm{H}\right\}$ NMR spectrum of Ru-dipp-Me-PC $\mathbf{y}_{3}$. 


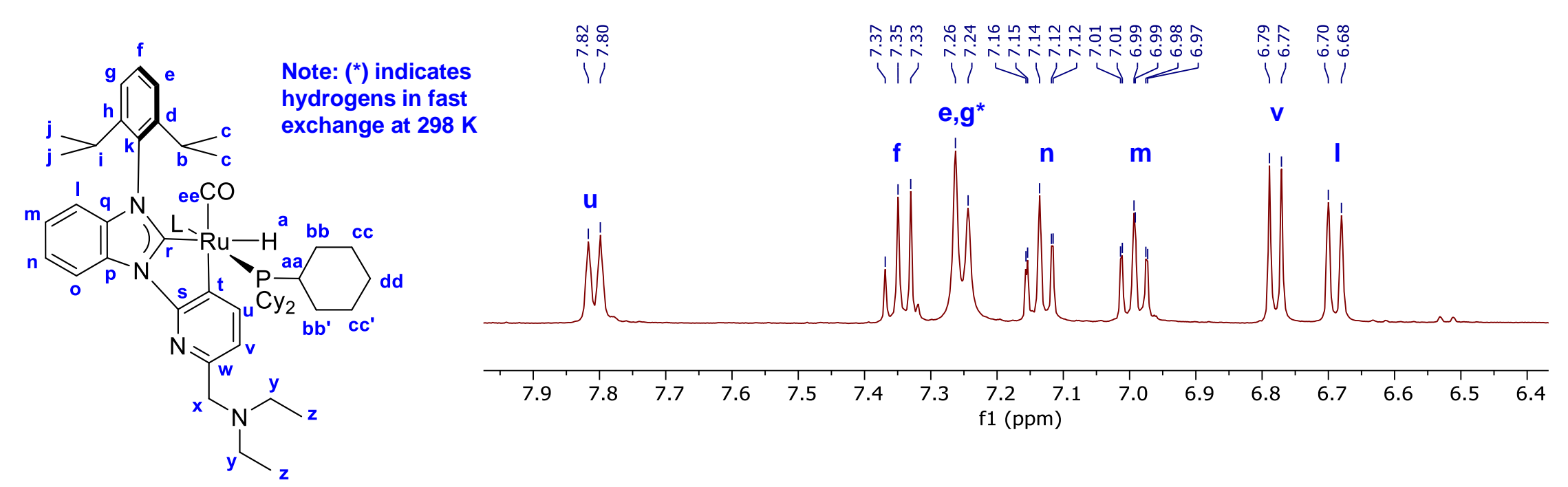

Ru-dipp-Et-PCy

$\mathrm{L}=\mathrm{PCy}_{3}$

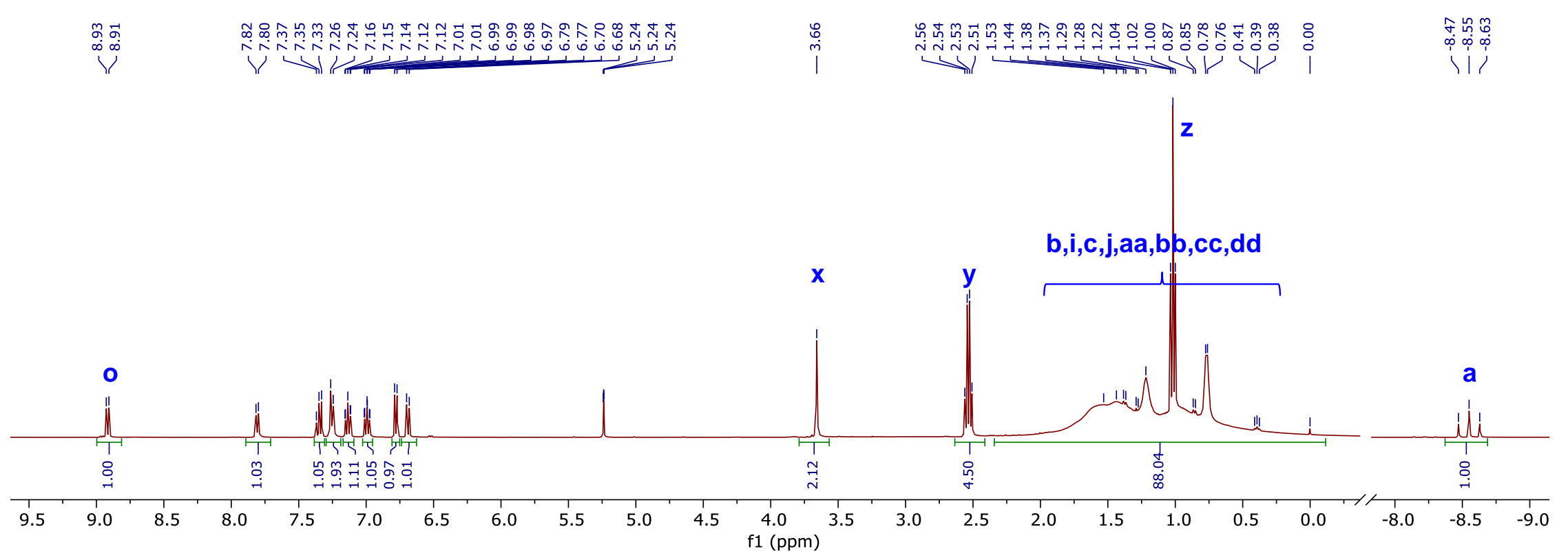

Figure S17. ${ }^{1} \mathrm{H}$ NMR spectrum of Ru-dipp-Et-PC $\mathbf{y}_{3}$. 

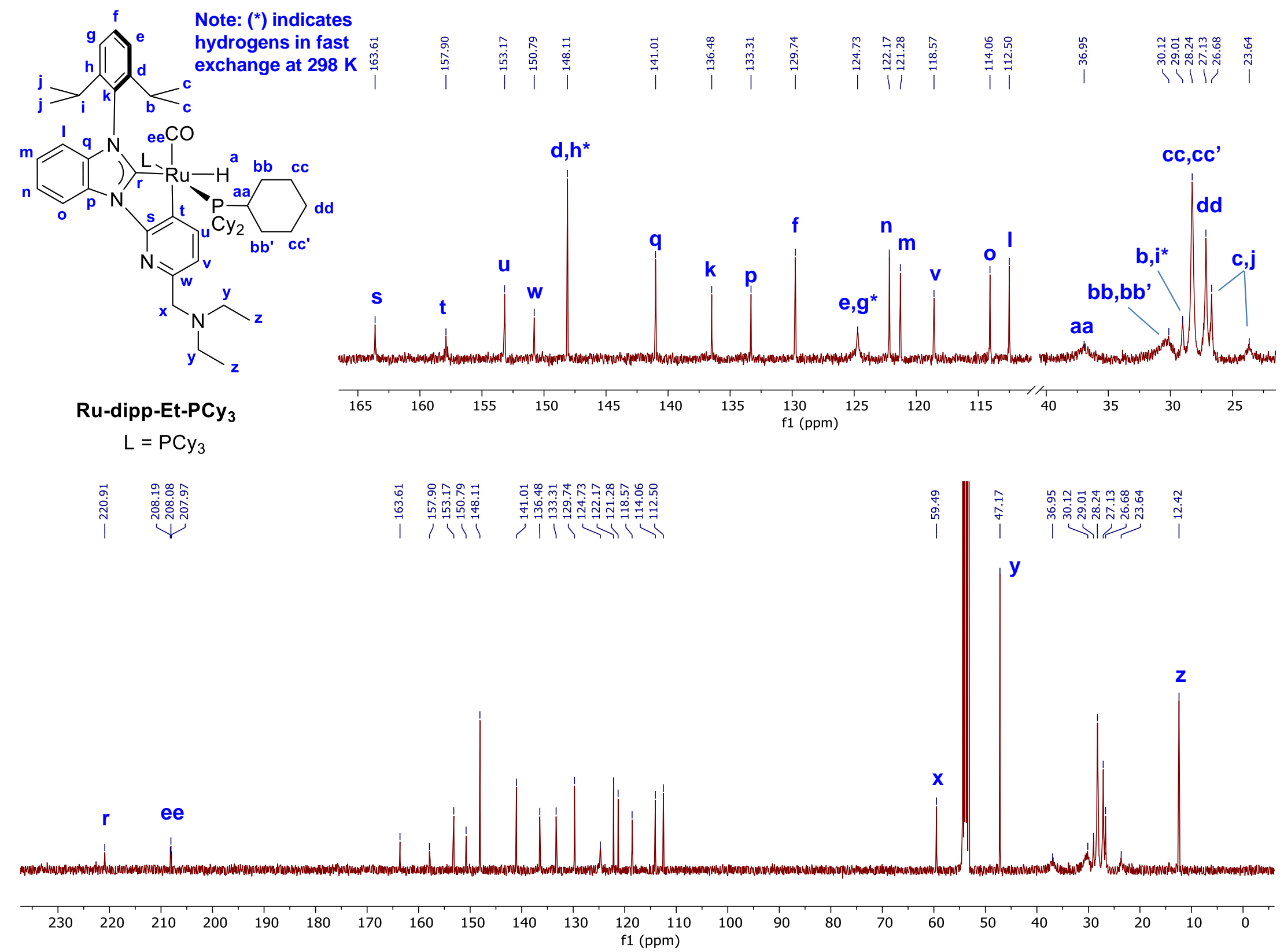

Figure S18. ${ }^{13} \mathrm{C}\left\{{ }^{1} \mathrm{H}\right\}$ NMR spectrum of Ru-dipp-Et-PCy. 


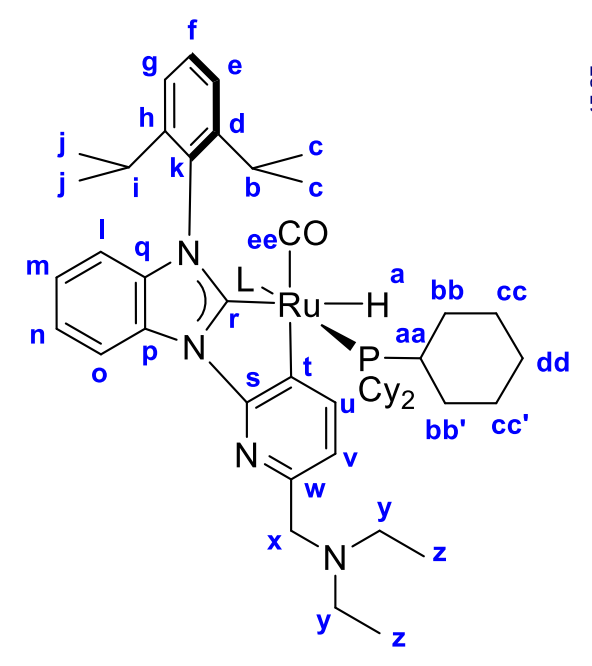

Ru-dipp-Et-PCy ${ }_{3}$

$\mathrm{L}=\mathrm{PCy}_{3}$

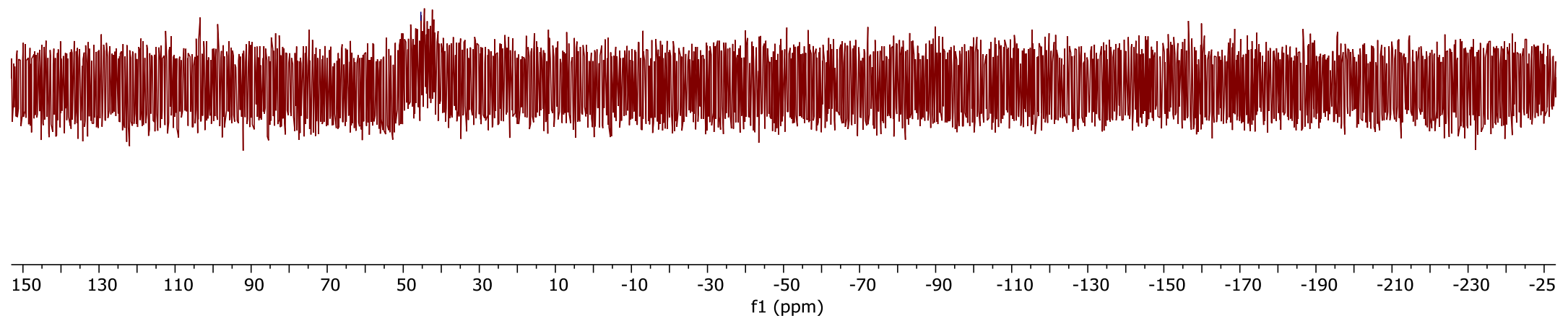

Figure S19. ${ }^{31} \mathrm{P}\left\{{ }^{1} \mathrm{H}\right\}$ NMR spectrum of Ru-dipp-Et-PCy $\mathbf{y}_{3}$. 


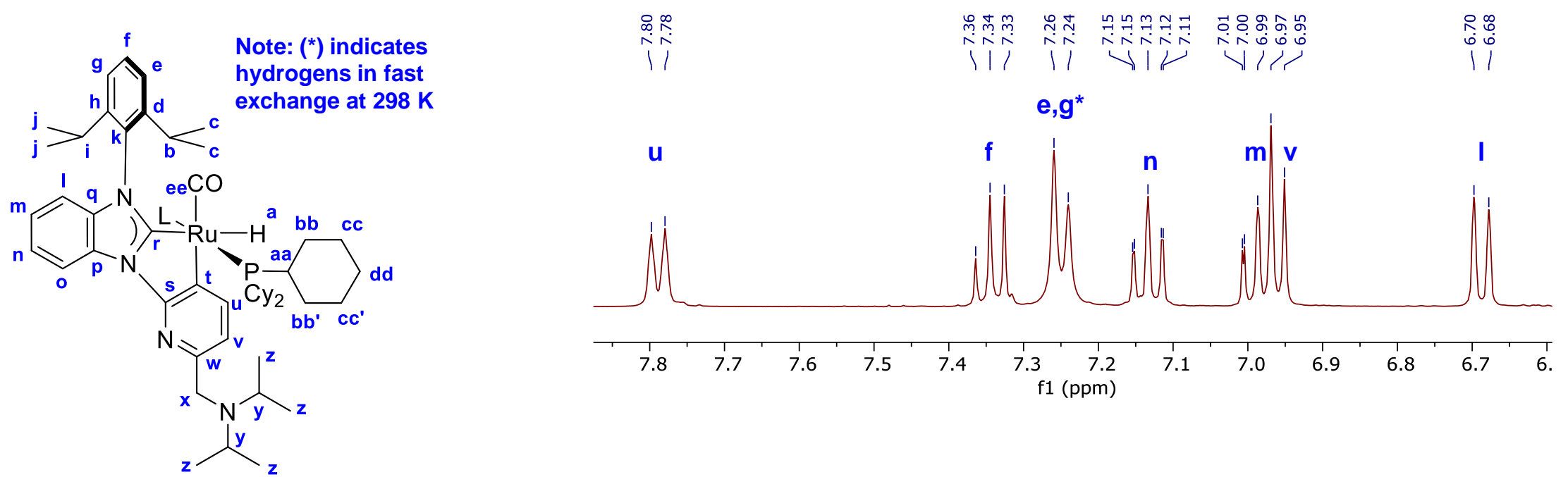

Ru-dipp-'Pr-PCy

$L=P C y_{3}$

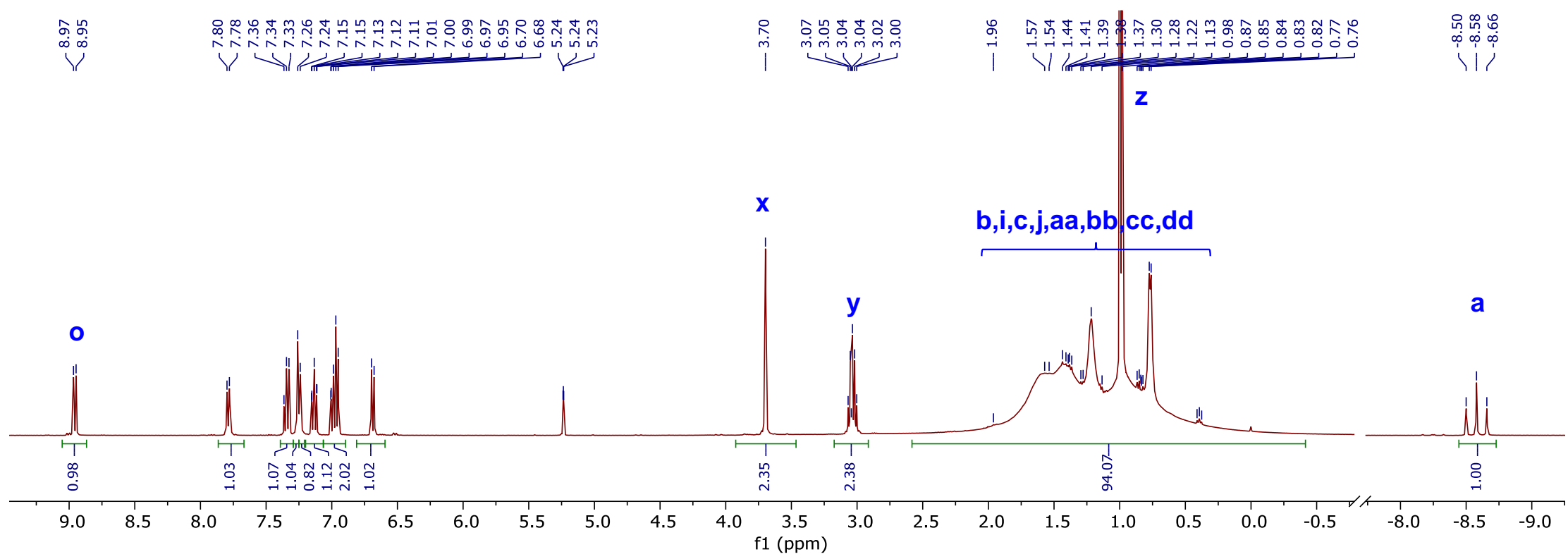

Figure S20. ${ }^{1} \mathrm{H}$ NMR spectrum of Ru-dipp-i'Pr-PCy. 


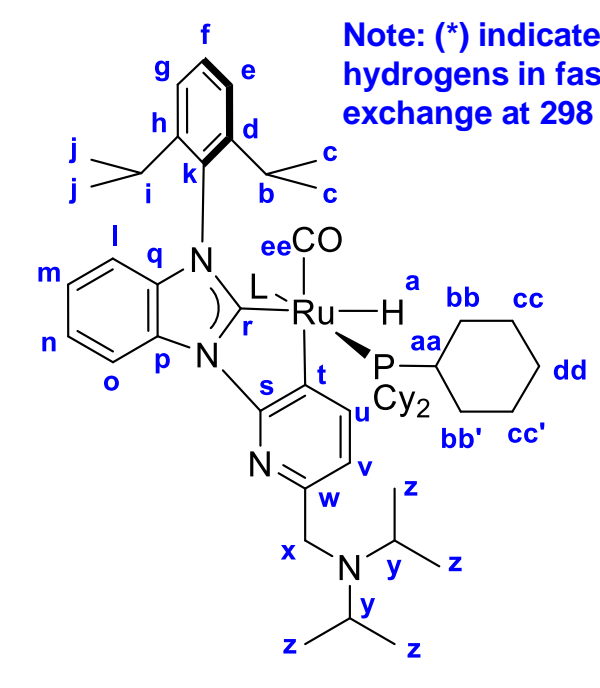

Ru-dipp-'Pr-PCy

$\mathrm{L}=\mathrm{PCy}_{3}$

1
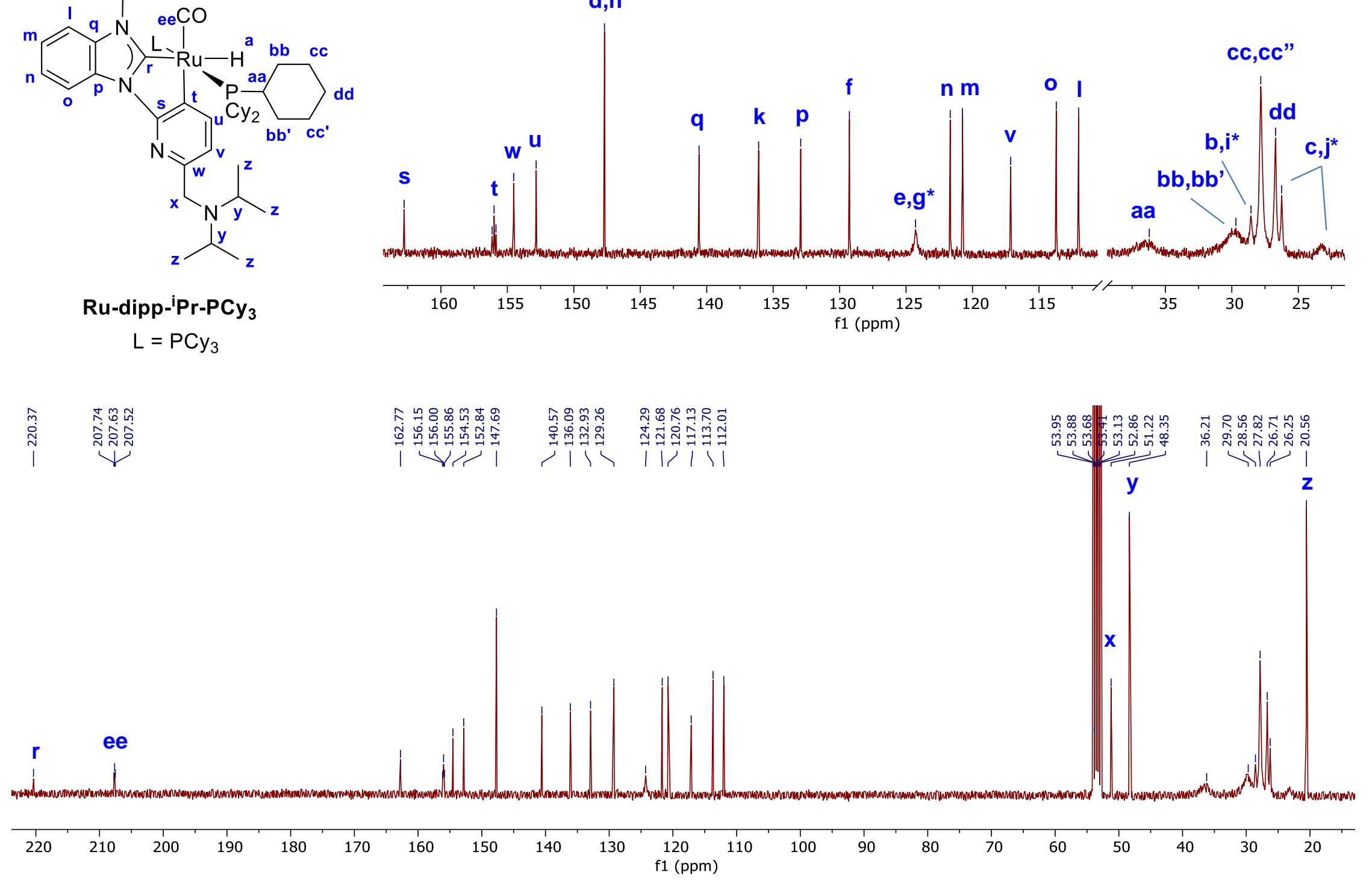

Figure S21. ${ }^{13} \mathrm{C}\left\{{ }^{1} \mathrm{H}\right\}$ NMR spectrum of Ru-dipp-'Pr-PC $\mathbf{y}_{\mathbf{3}}$. 


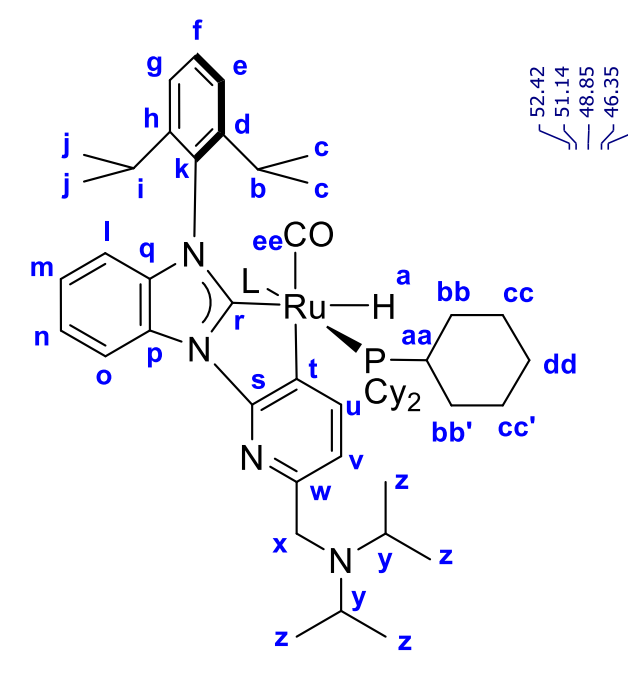

Ru-dipp-'Pr-PCy

$\mathrm{L}=\mathrm{PCy}_{3}$

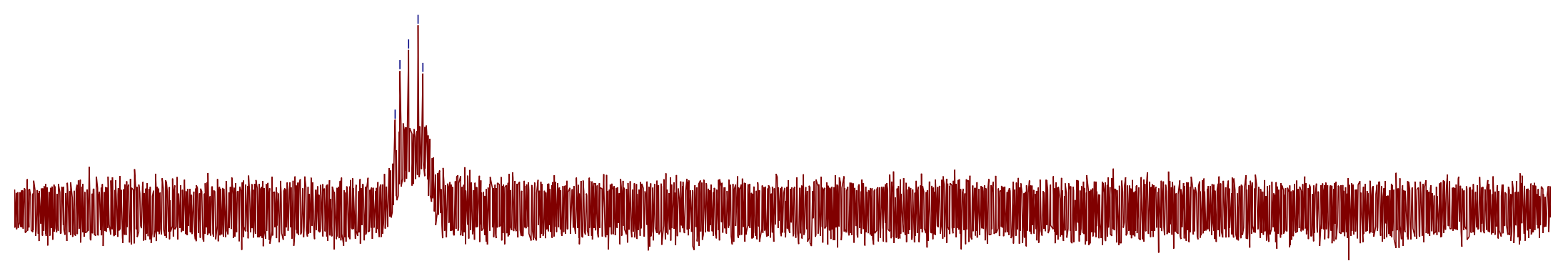

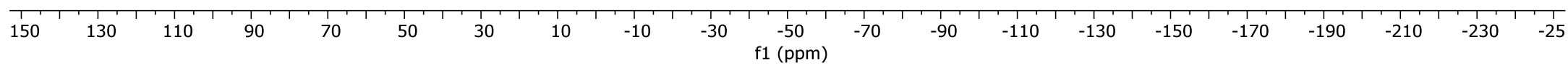

Figure S22. ${ }^{31} \mathrm{P}\left\{{ }^{1} \mathrm{H}\right\}$ NMR spectrum of Ru-dipp-'Pr-PCy. 